\title{
Curcumin alters gene expression-associated DNA damage, cell cycle, cell survival and cell migration and invasion in NCI-H460 human lung cancer cells in vitro
}

\author{
I-TSANG CHIANG ${ }^{1,2}$, WEI-SHU WANG ${ }^{3}$, HSIN-CHUNG LIU $^{4}$, SU-TSO YANG $^{5}$, \\ NOU-YING TANG ${ }^{6}$ and JING-GUNG CHUNG ${ }^{4,7}$
}

\author{
${ }^{1}$ Department of Radiation Oncology, National Yang-Ming University Hospital, Yilan 260; \\ ${ }^{2}$ Department of Radiological Technology, Central Taiwan University of Science and Technology, Taichung 40601; \\ ${ }^{3}$ Department of Internal Medicine, National Yang-Ming University Hospital, Yilan 260; \\ ${ }^{4}$ Department of Biological Science and Technology, China Medical University, Taichung 404; \\ ${ }^{5}$ Department of Radiology, China Medical University Hospital, Taichung 404; \\ ${ }^{6}$ Graduate Institute of Chinese Medicine, China Medical University, Taichung 404; \\ ${ }^{7}$ Department of Biotechnology, Asia University, Taichung 404, Taiwan, R.O.C.
}

Received March 31, 2015; Accepted June 26, 2015

DOI: $10.3892 / o r .2015 .4159$

\begin{abstract}
Lung cancer is the most common cause of cancer mortality and new cases are on the increase worldwide. However, the treatment of lung cancer remains unsatisfactory. Curcumin has been shown to induce cell death in many human cancer cells, including human lung cancer cells. However, the effects of curcumin on genetic mechanisms associated with these actions remain unclear. Curcumin $(2 \mu \mathrm{M})$ was added to NCI-H460 human lung cancer cells and the cells were incubated for $24 \mathrm{~h}$. Total RNA was extracted from isolated cells for cDNA synthesis, labeling, microarray hybridization and flour-labeled cDNA hybridized on chip. Localized concentrations of fluorescent molecules were detected and quantified using Expression Console software (Affymetrix) with default RMA parameters. GeneGo software was used for the key genes involved and their possible interaction pathways. The results showed that $\sim 170$ genes were significantly upregulated and 577 genes were significantly downregulated in curcumin-treated cells. Specifically, the up- and downregulated genes included $C C N E 2$, associated with DNA damage; ID3, associated with cell survival and 146 genes with a $>2$ - to 3 -fold change including the TP53INPI gene, associated with DNA damage; CDC6, CDCA5, TAKMIP2, CDK14, CDK5, CDCA76, $C D C 25 A, C D C 5 L$ and $S K P 2$, associated with cell cycle; the
\end{abstract}

Correspondence to: Professor Jing-Gung Chung, Department of Biological Science and Technology, China Medical University, 91 Hsueh-Shih Road, Taichung 404, Taiwan, R.O.C.

E-mail: jgchung@mail.cmu.edu.tw

Key words: curcumin, cDNA microarray, DNA damage, cell cycle, apoptosis, NCI-H460 cells
CARD6, IDI and ID2 genes, associated with cell survival and the BRMSIL, associated with cell migration and invasion. Additionally, 59 downregulated genes exhibited a $>4$-fold change, including the DDIT3 gene, associated with DNA damage; while 97 genes had a $>3$ - to 4 -fold change including the DDIT4 gene, associated with DNA damage; the CCPG1 gene, associated with cell cycle and 321 genes with a $>2$ - to 3-fold including the GADD45A and CGREF1 genes, associated with DNA damage; the $C C P G 1$ gene, associated with cell cycle, the TNFRSF10B, GAS5, TSSC1 and TNFRSF11B gene, associated with cell survival and the ARHAP29 and CADM2 genes, associated with cell migration and invasion. In conclusion, gene alterations provide information regarding the cytotoxic mechanism of curcumin at the genetic level and provide additional biomarkers or targets for the treatment of human lung cancer.

\section{Introduction}

Lung cancer, a leading cause of cancer-associated mortality wordwide, is one of the most aggressive human cancers and almost $80 \%$ of lung cancer-related deaths are non-small-cell lung cancer (NSCLC) $(1,2)$. Despite recent improvements in chemotherapy and molecular-targeted therapy in lung cancer patients, the outcome of lung cancer remains poor. Treatments can also cause serious side effects that affect quality of life of patients (3). Although there has been considerable progress in the diagnosis and treatment of lung cancer, the overall 5 -year survival rate of NSCLC patients remains $<15 \%$ (4). Thus, studies have focused on new anticancer drugs derived from natural products and attempted to improve the disadvantage of the treatment of lung cancer.

Curcumin, a component of turmeric, is derived from the rhizome of Curcuma longa. Previous findings have shown that curcumin has anticancer activities for various types of cancer in vitro and in vivo (5). Curcumin has been found to 
interfere with pathways that are overexpressed in cancer cells, such as NFKB, STAT3 and PI3K/Akt to induce cell death in tumors (6). Curcumin also acts as a chemoprotective agent (7-9). Intraperitoneally administered curcumin in rats was able to decrease brain-implanted glioblastomas in vivo (10). Additionally, curcumin induces autophagy by activating the AMPK signaling pathway in human lung adenocarcinoma cells (11). Curcumin inhibits migration and invasion of human lung cancer cells through inhibition of the Rac1/PAK1 signaling pathway and MMP-2 and MMP-9 (12). Recently, it was reported that curcumin significantly reduced the tumor growth of orthotopic human NSCLC xenografts and increased survival of treated athymic mice (13).

Cells maintain normal functions and survival via genome integrity. However, genome instability of cells causes genetic aberrations and is considered a hallmark of most types of cancer (14). After the presence of DNA damage, p53 protein can be activated to promote important gene expressions that are involved in cell cycle arrest, DNA repair and apoptosis (15). Thus, identification of genetic abnormalities in NSCLC has been used for the development of targeted therapeutic approaches in NSCLC treatment. Targeting tumors carrying mutations in EGFR or a fusion of the EML 4 and $A L K$ genes used clinically have been successful as first-line therapies in NSCLC (16-18). Therefore, it is imperative to identify gene expression or inhibition in NSCLC affected by test compounds or drugs to investigate the molecular mechanism to determine the function of these agents. Although curcumin has been demonstrated to induce cytotoxic effects on many human cancer cells, how associated total genes in human lung cancer cells (NSCLC) are affected by curcumin remains to be determined. Thus, we used cDNA microarray to investigate the altered gene expression in NCI-H460 cells following exposure to curcumin and the results indicated that curcumin altered certain gene expression associated with apoptosis.

\section{Materials and methods}

Chemicals and reagents. Curcumin and dimethylsulfoxide (DMSO) were obtained from Sigma Chemical Co. (St. Louis, MO, USA). Culture medium RPMI-1640, fetal bovine serum (FBS), 1\% L-glutamine, $100 \mathrm{U} / \mathrm{ml}$ penicillin and $100 \mu \mathrm{g} / \mathrm{ml}$ streptomycin were obtained from Gibco-BRL (Grand Island, NY, USA). Curcumin was dissolved in DMSO and stored at $-20^{\circ} \mathrm{C}$ prior to use.

Lung cancer cells. The NCI-H460 human NSCLC cell line was purchased from the Food Industry Research and Development Institute (Hsinchu, Taiwan). The cells were cultured in RPMI-1640 medium and supplemented with 10\% FBS, $1 \%$ L-glutamine and $1 \%$ penicillin/streptomycin (Invitrogen, Carlsbad, CA, USA) at $37^{\circ} \mathrm{C}$ in a humidified atmosphere of $95 \%$ air and $5 \% \mathrm{CO}_{2}$ air. The cells were split every 4 days to maintain exponential growth and were harvested with $0.025 \%$ trypsin and $0.52 \mathrm{mM}$ EDTA in phosphate-buffered saline (PBS), plated at required cell numbers and allowed to adhere for $\sim 24 \mathrm{~h}$ prior to treatment.

cDNA microarray assay used for gene expression in NCI-H460 cells following exposure to curcumin. NCI-H460 cells $\left(5 \times 10^{5}\right.$ cells $\left./ \mathrm{ml}\right)$ were kept in 12 -well plates with RPMI-1640 medium for $24 \mathrm{~h}$ and then incubated with or without $2 \mu \mathrm{M}$ of curcumin for $24 \mathrm{~h}$. After treatment, the cells were collected and total RNA from each sample was extracted using a Qiagen RNeasy Mini kit (Qiagen Inc., Valencia, CA, USA) as previously described (19). Total RNA from curcumin-treated and -untreated cells was quantified and used for cDNA synthesis, labeling and microarray hybridization, followed by flour-labeled cDNA hybridizing their complements on the chip (Affymetrix GeneChip Human Gene 1.0 ST array; Affymetrix, Santa Clara, CA, USA) as previously described (19). On the chip, the resulting localized concentrations of fluorescent molecules were detected and quantified (Asia BioInnovations Corporation, Taipei, Taiwan) and data were further analyzed using Expression Console software (Affymetrix) with default RMA parameters $(19,20)$. Up- or downregulated gene expressions was affected by DMC in NCI-H460 cells and a $\geq 2$-fold change was recorded and identified. Data were representative of three independent assays.

Statistical analysis. The results were representative of three assays. Differences between curcumin-treated and -untreated groups were presented up to 2 -fold changes, with + signifying upregulation and -, downregulation.

\section{Results}

Curcumin induces the up-and downregulated gene expression in NCI-H460 cells. The NCI-H460 cells were incubated with or without $2 \mu \mathrm{M}$ of curcumin and collected to extract total RNA. The cells were then used for cDNA microarray analysis and results are shown in Tables I and II. Table I indicated that 4 genes exhibited $>4$-fold change, 20 genes were $>3$ - to 4-fold including the CCNE2 gene, associated with DNA damage; the ID3 gene, associated with cell survival and 146 genes exhibited a $>2$ - to 3 -fold change, including the TP53INP1 gene, associated with DNA damage; the CDC6, CDCA5, TAKMIP2, CDK14, CDK5, CDCA76, CDC25A, $C D C 5 L$ and $S K P 2$ gene, associated with the cell cycle; the $C A R D 6, I D 1$ and ID2 genes, associated with cell survival and the BRMSIL gene, associated with cell migration and invasion.

Table II shows that 59 genes were downregulated with $>4$-fold change including the DDIT3 gene, associated with DNA damage, while 97 genes had a $>3$ - to 4-fold change, including the DDIT4 gene, associated with DNA damage; the CCPGl gene, associated with the cell cycle and 321 genes exhibited a $>2$ - to 3 -fold change including the GADD45A and $C G R E F 1$ genes, associated with DNA damage; the CCPG1 gene, associated with the cell cycle; the TNFRSF10B, GAS5, TSSC1 and TNFRSF11B genes, associated with cell survival; and the ARHAP29 and CADM2 genes, associated with cell migration and invasion.

Curcumin affects the gene expression scores as measured by GeneGo analysis program in NCI-H460 cells by the number of pathway networks. Curcumin-treated or -untreated cells were performed by cDNA microarray and then processed using GeneGo analysis. The results are shown in 
Table I. Representative genes of NCI-H460 cells upregulated following curcumin treatment.

\begin{tabular}{|c|c|c|}
\hline Fold-change & Gene symbol & mRNA description \\
\hline 5.64 & LOC 100506948 & $\begin{array}{l}\text { Uncharacterized LOC100506948; small nucleolar RNA, C/D box 116-28; } \\
115-26 ; 115-13 ; 115-7 ; 115-9 ; 115-11 ; 115-12 ; 115-29 ; 115-36 ; 115-39 ; 115-43\end{array}$ \\
\hline 4.57 & CYP1A1 & Cytochrome P450, family 1 , subfamily A \\
\hline 4.46 & TIPARP & TCDD-inducible poly(ADP-ribose) polymerase \\
\hline 4.03 & NAP1L2 & Nucleosome assembly protein 1-like 2 \\
\hline 3.98 & METTL7B & Methyltransferase-like 7B \\
\hline 3.79 & CCNE2 & Cyclin E2 \\
\hline 3.74 & DLX2 & Distal-less homeobox 2 \\
\hline 3.69 & TRNAK37P & Transfer RNA lysine 37 (anticodon CUU) pseudogene \\
\hline 3.62 & POLR2A & Polymerase (RNA) II (DNA directed) polypeptide A, $220 \mathrm{kDa}$ \\
\hline 3.56 & ID3 & Inhibitor of DNA binding 3, dominant-negative \\
\hline 3.49 & RN5S452 & RNA, $5 \mathrm{~S}$ ribosomal 452 \\
\hline 3.44 & SNRPN & $\begin{array}{l}\text { Uncharacterized LOC100506948; small nucleolar RNA, C/D box 116-28; } \\
115-26 ; 115-13 ; 115-7 ; 115-5 ; 115-9 ; 115-11 ; 115-12 ; 115-29\end{array}$ \\
\hline 3.40 & SNORD115-4 & Small nucleolar RNA, C/D box 115-4; 115-6; 115-42 \\
\hline 3.34 & RNU1-13P & RNA, U1 small nuclear 12, 13, pseudogene \\
\hline 3.29 & CLSPN & Claspin \\
\hline 3.26 & SNORD115-5 & $\begin{array}{l}\text { Small nucleolar RNA, C/D box } 115-5 ; 115-12 ; 115-9 ; 115-43 ; 115-36 \\
115-29 ; 115-11 ; 115-20\end{array}$ \\
\hline 3.20 & GUCY1B3 & Guanylate cyclase 1 , soluble, $\beta 3$ \\
\hline 3.20 & HSPA1A & Heat shock $70 \mathrm{kDa}$ protein $1 \mathrm{~A} ; 1 \mathrm{~B}$ \\
\hline 3.19 & SNORD115-6 & Small nucleolar RNA, C/D box 115-6; 115-42 \\
\hline 3.15 & SFN & Stratifin \\
\hline 3.09 & TUBB2A & Tubulin, $\beta 2$ A class IIa \\
\hline 3.05 & RNY4P15 & RNA, Ro-associated Y4 pseudogene 15 \\
\hline 3.03 & FLJ44896 & FLJ44896 protein \\
\hline 3.02 & PIK3R3 & Phosphoinositide-3-kinase, regulatory subunit $3(\gamma)$ \\
\hline 2.99 & CARD6 & Caspase recruitment domain family, member 6 \\
\hline 2.99 & TRNAU2 & Transfer RNA selenocysteine 2 (anticodon UCA) \\
\hline 2.96 & FOSB & FBJ murine osteosarcoma viral oncogene homolog B \\
\hline 2.94 & RNU1-10P & RNA, U1 small nuclear 10, pseudogene \\
\hline 2.93 & SAMD15 & Sterile $\alpha$ motif domain containing 15 \\
\hline 2.91 & FGD6 & FYVE, RhoGEF and PH domain containing 6 \\
\hline 2.87 & NAV1 & Neuron navigator 1 \\
\hline 2.85 & FAM171B & Family with sequence similarity 171 , member B \\
\hline 2.82 & VDR & Vitamin D $(1,25$-dihydroxyvitamin D3) receptor \\
\hline 2.81 & ORC1 & Origin recognition complex, subunit 1 \\
\hline 2.81 & DNAH14 & Dynein, axonemal, heavy chain 14 \\
\hline 2.77 & SNORD115-44 & Small nucleolar RNA, C/D box 115-44 \\
\hline 2.77 & GLULP4 & Glutamate-ammonia ligase (glutamine synthetase) pseudogene 4 \\
\hline 2.76 & LPCAT2 & Lysophosphatidylcholine acyltransferase 2 \\
\hline 2.75 & GTF2I & General transcription factor IIi \\
\hline 2.70 & ID1 & Inhibitor of DNA binding 1, dominant-negative helix-loop-helix protein \\
\hline 2.67 & HSPH1 & Heat shock 105/110 kDa protein 1 \\
\hline 2.58 & HIST1H3A & Histone cluster 1, H3a; H3f; H3b; H3h; H3j; H3g; H3i; H3e; H3c; H3d \\
\hline 2.57 & STOX1 & Storkhead box 1 \\
\hline 2.57 & RN5S183 & RNA, 5S ribosomal 183 \\
\hline 2.56 & CROT & Carnitine $O$-octanoyltransferase \\
\hline 2.56 & CDC6 & Cell division cycle 6 homolog (S. cerevisiae) \\
\hline 2.55 & IFIT3 & Interferon-induced protein with tetratricopeptide repeats 3 \\
\hline 2.54 & FOS & FBJ murine osteosarcoma viral oncogene homolog \\
\hline 2.52 & SLC29A3 & Solute carrier family 29 (nucleoside transporters), member 3 \\
\hline
\end{tabular}


Table I. Continued.

\begin{tabular}{|c|c|c|}
\hline Fold-change & Gene symbol & mRNA description \\
\hline 2.51 & PKI55 & DKFZp434H1419 \\
\hline 2.49 & HMGCS 1 & 3-Hydroxy-3-methylglutaryl-CoA synthase 1 (soluble) \\
\hline 2.45 & TACC1 & Transforming, acidic coiled-coil containing protein 1 \\
\hline 2.45 & DHFR & Dihydrofolate reductase \\
\hline 2.44 & ZNF480 & Zinc finger protein 480 \\
\hline 2.40 & KIF5C & Kinesin family member $5 \mathrm{C}$ \\
\hline 2.39 & DNAJA1 & DnaJ (Hsp40) homolog, subfamily A, member 1 \\
\hline 2.38 & PPP2R3A & Protein phosphatase 2 , regulatory subunit $B^{\prime}, \alpha$ \\
\hline 2.37 & HMGN3P1 & High mobility group nucleosomal binding domain 3 pseudogene 1 \\
\hline 2.37 & EML1 & Echinoderm microtubule-associated protein like 1 \\
\hline 2.33 & SRRT & Serrate RNA effector molecule homolog (Arabidopsis) \\
\hline 2.32 & DBR1 & Debranching enzyme homolog 1 (S. cerevisiae) \\
\hline 2.31 & JAKMIP2 & Janus kinase and microtubule interacting protein 2 \\
\hline 2.31 & CASC5 & Cancer susceptibility candidate 5 \\
\hline 2.30 & MFSD5 & Major facilitator superfamily domain containing 5 \\
\hline 2.30 & MCM4 & Minichromosome maintenance complex component 4 \\
\hline 2.29 & NEFL & Neurofilament, light polypeptide \\
\hline 2.29 & SGK3 & $\begin{array}{l}\text { Serum/glucocorticoid-regulated kinase family, member 3; } \\
\text { C8orf44-SGK3 readthrough }\end{array}$ \\
\hline 2.28 & NCOA5 & Nuclear receptor coactivator 5 \\
\hline 2.27 & MSRB 1 & Methionine sulfoxide reductase B1 \\
\hline 2.27 & BRMS1L & Breast cancer metastasis-suppressor 1-like \\
\hline 2.26 & NRG4 & Neuregulin 4 \\
\hline 2.26 & LOC 100653168 & Formin-1-like; formin 1 \\
\hline 2.26 & CDCA5 & Cell division cycle-associated 5 \\
\hline 2.26 & GINS3 & GINS complex subunit 3 (Psf3 homolog) \\
\hline 2.25 & LOC 100653119 & $\begin{array}{l}\text { Coiled-coil and } \mathrm{C} 2 \text { domain-containing protein } 2 \mathrm{~A} \text {-like; } \\
\text { coiled-coil and } \mathrm{C} 2 \text { domain containing } 2 \mathrm{~B}\end{array}$ \\
\hline 2.25 & LPHN2 & Latrophilin 2 \\
\hline 2.25 & KIF20A & Kinesin family member $20 \mathrm{~A}$ \\
\hline 2.25 & TMEM14A & Transmembrane protein $14 \mathrm{~A}$ \\
\hline 2.25 & HERC5 & HECT and RLD domain containing E3 ubiquitin protein ligase 5 \\
\hline 2.25 & TUBB4B & Tubulin, $\beta 4 \mathrm{~B}$ class IVb \\
\hline 2.25 & ANLN & Anillin, actin binding protein \\
\hline 2.24 & CKS2 & CDC28 protein kinase regulatory subunit 2 \\
\hline 2.23 & TSPYL2 & TSPY-like 2 \\
\hline 2.23 & RPA2 & Replication protein $\mathrm{A} 2,32 \mathrm{kDa}$ \\
\hline 2.22 & RNF38 & Ring finger protein 38 \\
\hline 2.22 & RFWD3 & Ring finger and WD repeat domain 3 \\
\hline 2.21 & GRK5 & G protein-coupled receptor kinase 5 \\
\hline 2.21 & ZNF107 & Zinc finger protein 107 \\
\hline 2.21 & SKA3 & Spindle and kinetochore-associated complex subunit 3 \\
\hline 2.21 & NAGK & $\mathrm{N}$-acetylglucosamine kinase \\
\hline 2.21 & CTR9 & Ctr9, Paf1/RNA polymerase II complex component, homolog (S. cerevisiae) \\
\hline 2.19 & $\mathrm{DIO} 2$ & Deiodinase, iodothyronine, type II \\
\hline 2.19 & AHSA1 & AHA1, activator of heat shock 90 kDa protein ATPase homolog 1 (yeast) \\
\hline 2.19 & C3orf14 & Chromosome 3 open reading frame 14 \\
\hline 2.18 & MARCH8 & Membrane-associated ring finger $(\mathrm{C} 3 \mathrm{HC} 4) 8, \mathrm{E} 3$ ubiquitin protein ligase \\
\hline 2.18 & LPAL2 & Lipoprotein, Lp(a)-like 2, pseudogene \\
\hline 2.18 & TUBB4B & Tubulin, $\beta 4 \mathrm{~B}$ class IVb \\
\hline 2.17 & GATA2 & GATA binding protein 2 \\
\hline 2.17 & PAR4 & Prader-Willi/Angelman region gene 4; small nucleolar RNA, C/D box 115-23 \\
\hline
\end{tabular}


Table I. Continued.

\begin{tabular}{|c|c|c|}
\hline Fold-change & Gene symbol & mRNA description \\
\hline 2.17 & TRIP13 & Thyroid hormone receptor interactor 13 \\
\hline 2.17 & MAPRE2 & Microtubule-associated protein, RP/EB family, member 2 \\
\hline 2.16 & CHST11 & Carbohydrate (chondroitin 4) sulfotransferase 11 \\
\hline 2.16 & SAMD9 & Sterile $\alpha$ motif domain containing 9 \\
\hline 2.16 & MT1X & Metallothionein $1 \mathrm{X}$ \\
\hline 2.16 & FNIP2 & Folliculin-interacting protein 2 \\
\hline 2.16 & TACC3 & Transforming, acidic coiled-coil containing protein 3 \\
\hline 2.15 & TERF1P2 & Telomeric repeat binding factor (NIMA-interacting) 1 pseudogene 2 \\
\hline 2.15 & ZNF611 & Zinc finger protein 611 \\
\hline 2.14 & C9orf78 & Chromosome 9 open reading frame 78 \\
\hline 2.14 & DHX9 & DEAH (Asp-Glu-Ala-His) box polypeptide 9 \\
\hline 2.13 & DHFR & Dihydrofolate reductase; dihydrofolate reductase pseudogene \\
\hline 2.13 & TMEM171 & Transmembrane protein 171 \\
\hline 2.12 & ID2 & Inhibitor of DNA binding 2, dominant negative helix-loop-helix protein \\
\hline 2.12 & CDK14 & Cyclin-dependent kinase 14 \\
\hline 2.12 & TRNASUP1 & Transfer RNA suppressor 1 (anticodon UUA) \\
\hline 2.11 & SMAD9 & SMAD family member 9 \\
\hline 2.11 & MCM2 & Minichromosome maintenance complex component 2 \\
\hline 2.11 & CT45A5 & $\begin{array}{l}\text { Cancer/testis antigen family } 45, \text { member A5; A } 2 \text {; A3; } \\
\text { A4 cancer/testis antigen family } 45, \text { member A } 1 \text {; A6 }\end{array}$ \\
\hline 2.11 & GDAP1 & Ganglioside induced differentiation associated protein 1 \\
\hline 2.11 & MCM10 & Minichromosome maintenance complex component 10 \\
\hline 2.11 & CABLES1 & Cdk5 and Abl enzyme substrate 1 \\
\hline 2.11 & CENPQ & Centromere protein $\mathrm{Q}$ \\
\hline 2.11 & CKAP2 & Cytoskeleton associated protein 2 \\
\hline 2.11 & FAM19A1 & Family with sequence similarity 19 [chemokine (C-C motif)-like], member A1 \\
\hline 2.11 & MEPCE & Methylphosphate capping enzyme \\
\hline 2.11 & REEP1 & Receptor accessory protein 1 \\
\hline 2.10 & SNORD115-32 & Small nucleolar RNA, C/D box 115-32 \\
\hline 2.10 & ATP6V0D1 & ATPase, $\mathrm{H}^{+}$transporting, lysosomal $38 \mathrm{kDa}, \mathrm{V} 0$ subunit $\mathrm{d} 1$ \\
\hline 2.10 & WDR6 & WD repeat domain 6 \\
\hline 2.10 & SNX10 & Sorting nexin 10 \\
\hline 2.10 & BRPF3 & Bromodomain and PHD finger containing, 3 \\
\hline 2.10 & FAM217B & Family with sequence similarity 217 , member B \\
\hline 2.10 & EML6 & Echinoderm microtubule-associated protein-like 6 \\
\hline 2.10 & SAFB2 & Scaffold attachment factor B2 \\
\hline 2.09 & TP53INP1 & Tumor protein p53 inducible nuclear protein 1 \\
\hline 2.09 & CPM & Carboxypeptidase $\mathrm{M}$ \\
\hline 2.09 & CDRT1 & CMT1A duplicated region transcript 1 \\
\hline 2.09 & CDCA7L & Cell division cycle associated 7-like \\
\hline 2.08 & SMAD6 & SMAD family member 6 \\
\hline 2.10 & SAFB2 & Scaffold attachment factor B2 \\
\hline 2.09 & TP53INP1 & Tumor protein p53 inducible nuclear protein 1 \\
\hline 2.09 & CPM & Carboxypeptidase $\mathrm{M}$ \\
\hline 2.09 & CDRT1 & CMT1A duplicated region transcript 1 \\
\hline 2.09 & CDCA7L & Cell division cycle associated 7-like \\
\hline 2.08 & SMAD6 & SMAD family member 6 \\
\hline 2.08 & CCDC138 & Coiled-coil domain containing 138 \\
\hline 2.07 & TUBB2A & Tubulin, $\beta 2$ A class IIa; tubulin, $\beta 2 \mathrm{~B}$ class IIb \\
\hline 2.07 & FEN1 & Flap structure-specific endonuclease 1 \\
\hline 2.06 & CYP1B1 & Cytochrome P450, family 1, subfamily B, polypeptide 1 \\
\hline 2.06 & $\mathrm{CDC} 25 \mathrm{~A}$ & Cell division cycle 25 homolog A (S.pombe) \\
\hline
\end{tabular}


Table I. Continued.

\begin{tabular}{|c|c|c|}
\hline Fold-change & Gene symbol & mRNA description \\
\hline 2.05 & WNK3 & WNK lysine deficient protein kinase 3 \\
\hline 2.05 & CXorf26 & Chromosome $\mathrm{X}$ open reading frame 26 \\
\hline 2.04 & TATDN2 & TatD DNase domain containing 2 \\
\hline 2.04 & ZNF840 & Zinc finger protein 840 \\
\hline 2.04 & EIF4EBP2 & Eukaryotic translation initiation factor $4 \mathrm{E}$ binding protein 2 \\
\hline 2.04 & PCYT2 & Phosphate cytidylyltransferase 2 , ethanolamine \\
\hline 2.03 & DUSP2 & Dual specificity phosphatase 2 \\
\hline 2.03 & CEP19 & Centrosomal protein $19 \mathrm{kDa}$ \\
\hline 2.03 & HMGN2 & High mobility group nucleosomal binding domain 2 \\
\hline 2.02 & DNAH6 & Dynein, axonemal, heavy chain 6 \\
\hline 2.02 & DYNLL1 & Dynein, light chain, LC8-type 1 \\
\hline 2.02 & NAT1 & $\mathrm{N}$-acetyltransferase 1 (arylamine $\mathrm{N}$-acetyltransferase) \\
\hline 2.02 & PPP2R5D & Protein phosphatase 2 , regulatory subunit $B^{\prime}, \delta$ \\
\hline 2.02 & DPYSL3 & Dihydropyrimidinase-like 3 \\
\hline 2.02 & PCYT2 & Phosphate cytidylyltransferase 2 , ethanolamine \\
\hline 2.02 & SHCBP1 & SHC SH2-domain binding protein 1 \\
\hline 2.02 & CDC5L & CDC5 cell division cycle 5-like (S. pombe) \\
\hline 2.02 & CDKN2C & Cyclin-dependent kinase inhibitor 2C (p18, inhibits CDK4) \\
\hline 2.02 & FRMD3 & FERM domain containing 3 \\
\hline 2.02 & SKP2 & S-phase kinase-associated protein 2, E3 ubiquitin protein ligase \\
\hline 2.01 & TRNAP28P & Transfer RNA proline 28 (anticodon AGG) pseudogene \\
\hline 2.01 & LOC100129648 & Uncharacterized LOC100129648 \\
\hline 2.01 & NXF1 & Nuclear RNA export factor 1 \\
\hline 2.01 & ADI1 & Acireductone dioxygenase 1 \\
\hline 2.01 & $\mathrm{CFB}$ & Complement factor B \\
\hline 2.01 & ZNF483 & Zinc finger protein 483 \\
\hline
\end{tabular}

Figs. 1-3 for top, second and third scores, respectively. The analysis results were mapped on the processes as presented in possible signal effects. Red indicated upregulated genes and blue, the downregulated genes. Circles indicated different intensities indicating different enhancing or inhibitions in curcumin-treated NCI-H460 cells.

\section{Discussion}

It has been demonstrated that curcumin induced biological activities including anticancer function in vitro and in vivo. However, how curcumin affects gene expression and the associated signaling pathways in human lung cancer cells remains to be determined. Thus, we investigated the up- and downregulation of associated genes with cell cycle, DNA damage, cell survival and cell migration and invasion in NCI H460 cells. In the present study, we found several genes involved DNA damage and repair were increased, such as cyclin E2 (CCNE2), which was increased 3.79-fold. It has also been reported that following cell induction DNA damage led to cell cycle arrest (21). The tumor protein p53 inducible nuclear protein 1 (TP53INP1) was increased 2.09-fold and after DNA damage the p53 protein expression was also increased (22).

The results showed that the TP53INP1 gene, associated with DNA damage was increased 2.09-fold, the CDC6, TAKMIP2,
CDCA5, CDK14, CDK5, CDCA76, CDC25A, CDC5L and $S K P 2$, associated with cell cycle were increased 2.56-, 2.31-, 2.26-, 2.12-, 2.11-, 2.09-, 2.06-, 2.02- and 2.02-fold, respectively, associated with cell cycle, while the CARD6, IDI and ID2 genes, associated with cell survival were increased 2.99-, 2.70- and 2.12-fold. It was also found that the BRMS1L gene, associated with cell migration and invasion, was increased 2.27-fold. It was reported that tumor protein 53-induced nuclear protein 1 (TP53INP1) is a stress-induced p53-target gene function that represses tumorigenesis (23). The cell cycle-dependent centrosomal localization of Cdc6 in the $\mathrm{S}$ and $\mathrm{G} 2$ phases is considered to have a novel function of Cdc6 in centrosomes (24). CDCA5 and CDK5 have been found to play an important role in the cell cycle (25). It was reported that the mitotic CDK14/cyclin Y complex promotes Wnt signaling (26). Moreover, Cdc25 is known to be involved cell cycle regulation (27). Caspase recruitment domain 6 (CARD6), a microtubule-interacting protein, belongs to the CARD family (28). CARD6 may be a novel target for the treatment of pathological cardiac hypertrophy and failure (29). ID (inhibitor of differentiation and DNA binding) comprises the ID1, ID2, ID3 and ID4 proteins, which are involved in cancer progression (30). ID1 (inhibitor of differentiation and DNA binding 1) was reported to strongly correlate with various types of tumors, including lung cancer $(31,32)$. 
Table II. Representative genes of NCI-H460 cells those were upregulated by curcumin treatment.

\begin{tabular}{|c|c|c|}
\hline Fold-change & Gene symbol & mRNA description \\
\hline-2.01 & HORMAD1 & HORMA domain containing 1 \\
\hline-2.01 & EGFR & Epidermal growth factor receptor \\
\hline-2.01 & VTN & Vitronectin; SEBOX homeobox \\
\hline-2.01 & PGAP1 & Post-GPI attachment to proteins 1 \\
\hline-2.01 & ZAK & Sterile $\alpha$ motif and leucine zipper containing kinase AZK \\
\hline-2.01 & PLOD1 & Procollagen-lysine, 2-oxoglutarate 5-dioxygenase 1 \\
\hline-2.01 & HPCAL1 & Hippocalcin-like 1 \\
\hline-2.01 & ALS2CR8 & Amyotrophic lateral sclerosis 2 (juvenile) chromosome region, candidate 8 \\
\hline-2.01 & RPS6KA2 & Ribosomal protein S6 kinase, 90 kDa, polypeptide 2 \\
\hline-2.01 & ITPR3 & Inositol 1,4,5-trisphosphate receptor, type 3 \\
\hline-2.01 & LEPRE1 & Leucine proline-enriched proteoglycan (leprecan) 1 \\
\hline-2.01 & FJX1 & Four jointed box 1 (Drosophila) \\
\hline-2.02 & GALC & Galactosylceramidase \\
\hline-2.02 & TNFRSF11B & Tumor necrosis factor receptor superfamily, member $11 \mathrm{~b}$ \\
\hline-2.02 & DENND3 & DENN/MADD domain containing 3 \\
\hline-2.02 & SORT1 & Sortilin 1 \\
\hline-2.02 & IGFBP7 & Insulin-like growth factor binding protein 7 \\
\hline-2.02 & IGF2BP2 & Insulin-like growth factor 2 mRNA binding protein 2 \\
\hline-2.02 & TAS2R31 & Taste receptor, type 2 , member 31 ; taste receptor, type 2 , member 45 \\
\hline-2.02 & LMBRD2 & LMBR1 domain containing 2 \\
\hline-2.02 & SNORD77 & Small nucleolar RNA, C/D box 77 \\
\hline-2.02 & C6orf120 & Chromosome 6 open reading frame 120 \\
\hline-2.02 & FAM188A & Family with sequence similarity 188 , member A \\
\hline-2.02 & RBCK1 & RanBP-type and C3HC4-type zinc finger containing 1 \\
\hline-2.02 & FHDC1 & FH2 domain containing 1 \\
\hline-2.03 & DYNC1H1 & Dynein, cytoplasmic 1 , heavy chain 1 \\
\hline-2.03 & ANKH & Ankylosis, progressive homolog (mouse) \\
\hline-2.03 & TSSC1 & Tumor-suppressing subtransferable candidate 1 \\
\hline-2.03 & CLYBL & Citrate lyase $\beta$-like \\
\hline-2.03 & GAS5 & Growth arrest-specific 5 (non-protein coding); small nucleolar RNA, C/D box 80 \\
\hline-2.03 & CEBPG & CCAAT/enhancer binding protein $(\mathrm{C} / \mathrm{EBP}), \gamma$ \\
\hline-2.04 & BCKDHA & Branched chain keto acid dehydrogenase E1, $\alpha$ polypeptide \\
\hline-2.04 & CCNB1IP1 & Cyclin B1 interacting protein 1, E3 ubiquitin protein ligase \\
\hline-2.04 & TMEM67 & Transmembrane protein 67 \\
\hline-2.02 & RBCK1 & RanBP-type and C3HC4-type zinc finger containing 1 \\
\hline-2.02 & FAM27E3 & Family with sequence similarity 27, member E3; E2 \\
\hline-2.02 & FHDC1 & FH2 domain containing 1 \\
\hline-2.03 & DYNC1H1 & Dynein, cytoplasmic 1 , heavy chain 1 \\
\hline-2.03 & SNORD49B & $\begin{array}{l}\text { Small nucleolar RNA, C/D box 49B; box } 65 ; \text { C17orf76 antisense RNA } 1 \\
\text { (non-protein coding); small nucleolar RNA, C/D box } 49 \mathrm{~A}\end{array}$ \\
\hline-2.03 & ANKH & Ankylosis, progressive homolog (mouse) \\
\hline-2.03 & TSSC1 & Tumor-suppressing subtransferable candidate 1 \\
\hline-2.03 & CLYBL & Citrate lyase $\beta$-like \\
\hline-2.03 & GAS5 & Growth arrest-specific 5 (non-protein coding); small nucleolar RNA, C/D box 80 \\
\hline-2.02 & FHDC1 & FH2 domain containing 1 \\
\hline-2.03 & DYNC1H1 & Dynein, cytoplasmic 1 , heavy chain 1 \\
\hline-2.03 & SNORD49B & $\begin{array}{l}\text { Small nucleolar RNA, C/D box 49B; 65; C17orf76 antisense RNA } 1 \\
\text { (non-protein coding); small nucleolar RNA, C/D box 49A }\end{array}$ \\
\hline-2.03 & TSSC1 & Tumor-suppressing subtransferable candidate 1 \\
\hline-2.03 & CLYBL & Citrate lyase $\beta$-like \\
\hline-2.03 & GAS5 & Growth arrest-specific 5 (non-protein coding); small nucleolar RNA, C/D box 80 \\
\hline-2.03 & CEBPG & CCAAT/enhancer binding protein $(\mathrm{C} / \mathrm{EBP}), \gamma$ \\
\hline
\end{tabular}


Table II. Continued.

\begin{tabular}{|c|c|c|}
\hline Fold-change & Gene symbol & mRNA description \\
\hline-2.04 & BCKDHA & Branched chain keto acid dehydrogenase E1, $\alpha$ polypeptide \\
\hline-2.04 & CCNB1IP1 & Cyclin B1 interacting protein 1, E3 ubiquitin protein ligase \\
\hline-2.04 & LCN1 & Lipocalin 1 \\
\hline-2.04 & TMEM67 & Transmembrane protein 67 \\
\hline-2.04 & PLD3 & Phospholipase D family, member 3 \\
\hline-2.04 & FAM27E3 & $\begin{array}{l}\text { Family with sequence similarity } 27 \text {, member E3; family with sequence similarity } 27 \text {, } \\
\text { member E2 }\end{array}$ \\
\hline-2.04 & ABCA2 & ATP-binding cassette, sub-family A (ABC1), member 2 \\
\hline-2.05 & PBX1 & Pre-B-cell leukemia homeobox 1 \\
\hline-2.05 & C11orf54 & Chromosome 11 open reading frame 54 \\
\hline-2.05 & KRTAP5-5 & Keratin-associated protein $5-5$ \\
\hline-2.05 & RAP2B & RAP2B, member of RAS oncogene family \\
\hline 2.05 & PLK2 & Polo-like kinase 2 \\
\hline-2.05 & SLC9A3 & Solute carrier family 9 , subfamily A (NHE3, cation proton antiporter 3 ), member 3 \\
\hline-2.05 & ARSG & Arylsulfatase G \\
\hline-2.06 & CADM2 & Cell adhesion molecule 2 \\
\hline-2.06 & SCD & Stearoyl-CoA desaturase ( $\delta$-9-desaturase) \\
\hline 2.05 & PLK2 & Polo-like kinase 2 \\
\hline-2.05 & SLC9A3 & Solute carrier family 9, subfamily A (NHE3, cation proton antiporter 3 ), member 3 \\
\hline-2.05 & ARSG & Arylsulfatase G \\
\hline-2.06 & CADM2 & Cell adhesion molecule 2 \\
\hline-2.06 & SLPI & Secretory leukocyte peptidase inhibitor \\
\hline-2.06 & RND3 & Rho family GTPase 3 \\
\hline-2.06 & CD9 & CD9 molecule; uncharacterized LOC100653288 \\
\hline-2.06 & PLOD2 & Procollagen-lysine, 2-oxoglutarate 5-dioxygenase 2 \\
\hline-2.06 & GPC6 & Glypican 6 \\
\hline-2.06 & RASSF8 & Ras association (RalGDS/AF-6) domain family (N-terminal) member 8 \\
\hline-2.06 & MEST & Mesoderm-specific transcript homolog (mouse) \\
\hline-2.06 & SIAE & Sialic acid acetylesterase \\
\hline-2.06 & CSGALNACT2 & Chondroitin sulfate $\mathrm{N}$-acetylgalactosaminyltransferase 2 \\
\hline-2.06 & JHDM1D & Jumonji C domain containing histone demethylase 1 homolog D (S. cerevisiae) \\
\hline-2.06 & ASAH1 & $\mathrm{N}$-acylsphingosine amidohydrolase (acid ceramidase) 1 \\
\hline-2.07 & P2RX4 & Purinergic receptor P2X, ligand-gated ion channel, 4 \\
\hline-2.07 & RBM24 & RNA binding motif protein 24 \\
\hline-2.07 & GPR108 & G protein-coupled receptor 108 \\
\hline-2.07 & ACTR1B & ARP1 actin-related protein 1 homolog $B$, centractin $\beta$ (yeast) \\
\hline-2.07 & CTPS1 & CTP synthase 1 \\
\hline-2.07 & RNF41 & Ring finger protein 41 \\
\hline-2.07 & SLC1A4 & Solute carrier family 1 (glutamate/neutral amino acid transporter), member 4 \\
\hline-2.08 & $\mathrm{BCHE}$ & Butyrylcholinesterase \\
\hline-2.08 & GPT2 & Glutamic pyruvate transaminase (alanine aminotransferase) 2 \\
\hline-2.08 & COL12A1 & Collagen, type XII, $\alpha 1$ \\
\hline-2.08 & ARG2 & Arginase, type II \\
\hline-2.08 & HFM1 & HFM1, ATP-dependent DNA helicase homolog (S. cerevisiae) \\
\hline-2.08 & NOX1 & NADPH oxidase 1 \\
\hline-2.08 & SNX14 & Sorting nexin 14 \\
\hline-2.08 & CD109 & CD109 molecule \\
\hline-2.09 & CNTNAP3 & Contactin-associated protein-like 3; contactin-associated protein-like 3B \\
\hline-2.09 & MCL1 & Myeloid cell leukemia sequence 1 (BCL2-related) \\
\hline-2.09 & L1CAM & L1 cell adhesion molecule \\
\hline-2.09 & PART1 & Prostate androgen-regulated transcript 1 (non-protein coding) \\
\hline-2.09 & TCF4 & Transcription factor 4 \\
\hline
\end{tabular}


Table II. Continued.

\begin{tabular}{|c|c|c|}
\hline Fold-change & Gene symbol & mRNA description \\
\hline-2.09 & IGF1R & Insulin-like growth factor 1 receptor \\
\hline-2.09 & LRP12 & Low-density lipoprotein receptor-related protein 12 \\
\hline-2.09 & SLC50A1 & Solute carrier family 50 (sugar transporter), member 1 \\
\hline-2.09 & GLDN & Gliomedin \\
\hline-2.10 & CYFIP2 & Cytoplasmic FMR1 interacting protein 2 \\
\hline-2.10 & SNORD116@ & Small nucleolar RNA, C/D box 116 cluster; small nucleolar RNA, C/D box 116-21 \\
\hline-2.10 & TCEA1 & Transcription elongation factor A (SII), 1 \\
\hline-2.10 & NCOR2 & Nuclear receptor corepressor 2 \\
\hline-2.10 & EFHC1 & EF-hand domain (C-terminal) containing 1 \\
\hline-2.10 & SLC11A2 & Solute carrier family 11 (proton-coupled divalent metal ion transporters), member 2 \\
\hline-2.11 & CDH11 & Cadherin 11, type 2 , OB-cadherin (osteoblast) \\
\hline-2.11 & NOSTRIN & Nitric oxide synthase trafficker \\
\hline-2.11 & EDEM1 & ER degradation enhancer, mannosidase $\alpha$-like 1 \\
\hline-2.11 & ALDOC & Aldolase $\mathrm{C}$, fructose-bisphosphate \\
\hline-2.11 & NEDD1 & Neural precursor cell expressed, developmentally downregulated 1 \\
\hline-2.11 & SARS & Seryl-tRNA synthetase \\
\hline-2.11 & RN5S385 & RNA, 5S ribosomal 385 \\
\hline-2.11 & RAPGEF4 & Rap guanine nucleotide exchange factor (GEF) 4 \\
\hline-2.11 & ENOSF1 & Enolase superfamily member 1 \\
\hline-2.12 & PRKACB & Protein kinase, cAMP-dependent, catalytic, $\beta$ \\
\hline-2.12 & CSNK1E & Casein kinase $1, \varepsilon$ \\
\hline-2.12 & ALG13 & Asparagine-linked glycosylation 13 homolog (S. cerevisiae) \\
\hline-2.12 & DEPDC1 & DEP domain containing 1 \\
\hline-2.12 & IGFBP3 & Insulin-like growth factor binding protein 3 \\
\hline-2.12 & UACA & Uveal autoantigen with coiled-coil domains and ankyrin repeats \\
\hline-2.12 & COL3A1 & Collagen, type III, $\alpha 1$ \\
\hline-2.12 & IVNS1ABP & Influenza virus NS1A binding protein \\
\hline-2.13 & EPB41L3 & Erythrocyte membrane protein band 4.1-like 3 \\
\hline-2.13 & STEAP1B & STEAP family member $1 \mathrm{~B}$ \\
\hline-2.13 & SOCS3 & Suppressor of cytokine signaling 3 \\
\hline-2.13 & MIR186 & microRNA 186 \\
\hline-2.13 & GFPT1 & Glutamine-fructose-6-phosphate transaminase 1 \\
\hline-2.14 & MORC4 & MORC family CW-type zinc finger 4 \\
\hline-2.14 & ANKRD28 & Ankyrin repeat domain 28 \\
\hline-2.14 & MTMR11 & Myotubularin-related protein 11 \\
\hline-2.14 & HLA-F & Major histocompatibility complex, class I, F \\
\hline-2.14 & PIGK & Phosphatidylinositol glycan anchor biosynthesis, class $\mathrm{K}$ \\
\hline-2.14 & NUCB1 & Nucleobindin 1 \\
\hline-2.14 & ERAP1 & Endoplasmic reticulum aminopeptidase 1 \\
\hline-2.14 & PAN2 & PAN2 poly(A) specific ribonuclease subunit homolog (S. cerevisiae) \\
\hline-2.14 & SYCP2L & Synaptonemal complex protein 2-like \\
\hline-2.15 & ADAMTS1 & ADAM metallopeptidase with thrombospondin type 1 motif, 1 \\
\hline-2.15 & IL1A & Interleukin $1, \alpha$ \\
\hline-2.15 & ARHGAP29 & Rho GTPase-activating protein 29 \\
\hline-2.15 & C18orf54 & Chromosome 18 open reading frame 54 \\
\hline-2.15 & ENC1 & Ectodermal-neural cortex 1 (with BTB-like domain) \\
\hline-2.15 & HSPG2 & Heparan sulfate proteoglycan 2 \\
\hline-2.16 & MIR492 & microRNA 492; keratin 19 pseudogene 2 \\
\hline-2.16 & DNASE2 & Deoxyribonuclease II, lysosomal \\
\hline-2.16 & ABCA1 & ATP-binding cassette, sub-family A (ABC1), member 1 \\
\hline-2.16 & MAML3 & Mastermind-like 3 (Drosophila) \\
\hline-2.16 & SNORA27 & Small nucleolar RNA, H/ACA box 27; 102; ribosomal protein L21 \\
\hline
\end{tabular}


Table II. Continued.

\begin{tabular}{|c|c|c|}
\hline Fold-change & Gene symbol & mRNA description \\
\hline-2.16 & GSTT1 & Glutathione S-transferase $\theta 1$ \\
\hline-2.17 & DCLK2 & Doublecortin-like kinase 2 \\
\hline-2.17 & PPP2R3C & Protein phosphatase 2 , regulatory subunit $\mathrm{B}^{\prime}, \gamma$ \\
\hline-2.17 & MARCH1 & Membrane-associated ring finger $(\mathrm{C} 3 \mathrm{HC} 4) 1, \mathrm{E} 3$ ubiquitin protein ligase \\
\hline-2.17 & SPG11 & Spastic paraplegia 11 (autosomal recessive) \\
\hline-2.17 & ANK3 & Ankyrin 3, node of Ranvier (ankyrin G) \\
\hline-2.17 & LINGO2 & Leucine rich repeat and Ig domain containing 2 \\
\hline-2.17 & TNFRSF10B & Tumor necrosis factor receptor superfamily, member $10 \mathrm{~b}$ \\
\hline-2.17 & SRPX2 & Sushi-repeat containing protein, X-linked 2 \\
\hline-2.17 & ZNF41 & Zinc finger protein 41 \\
\hline-2.17 & MAN2C1 & Mannosidase, $\alpha$, class $2 \mathrm{C}$, member 1 \\
\hline-2.18 & PDE7B & Phosphodiesterase 7B \\
\hline-2.18 & EPAS1 & Endothelial PAS domain protein 1; uncharacterized LOC100652809 \\
\hline-2.18 & FN1 & Fibronectin 1 \\
\hline-2.18 & ETV4 & Ets variant 4 \\
\hline-2.18 & CTSO & Cathepsin $\mathrm{O}$ \\
\hline-2.18 & MGAT4C & $\begin{array}{l}\text { Mannosyl ( } \alpha-1,3-) \text {-glycoprotein } \\
\beta-1,4-N \text {-acetylglucosaminyltransferase, isozyme C (putative) }\end{array}$ \\
\hline-2.18 & R3HDM2 & $\mathrm{R} 3 \mathrm{H}$ domain containing 2 \\
\hline-2.18 & AMDHD1 & Amidohydrolase domain containing 1 \\
\hline-2.18 & SNORA68 & Small nucleolar RNA, H/ACA box 68 \\
\hline-2.18 & SLC7A1 & Solute carrier family 7 (cationic amino acid transporter, $\mathrm{y}^{+}$system), member 1 \\
\hline-2.19 & NID2 & Nidogen 2 (osteonidogen) \\
\hline-2.19 & LXN & Latexin \\
\hline-2.19 & CTIF & CBP80/20-dependent translation initiation factor \\
\hline-2.19 & TRNAG36 & $\begin{array}{l}\text { Transfer RNA glycine } 36 \text { (anticodon CCC); transfer RNA valine } 34 \\
\text { (anticodon CAC) pseudogene }\end{array}$ \\
\hline-2.19 & ST6GAL1 & ST6 $\beta$-galactosamide $\alpha$-2,6-sialyltranferase 1 \\
\hline-2.19 & SCARNA23 & Small Cajal body-specific RNA 23 \\
\hline-2.19 & DPYD & Dihydropyrimidine dehydrogenase \\
\hline-2.19 & DDR2 & Discoidin domain receptor tyrosine kinase 2 \\
\hline-2.18 & FN1 & Fibronectin 1 \\
\hline-2.18 & ETV4 & Ets variant 4 \\
\hline-2.18 & CTSO & Cathepsin $\mathrm{O}$ \\
\hline-2.18 & MGAT4C & $\begin{array}{l}\text { Mannosyl ( } \alpha-1,3-) \text {-glycoprotein } \beta-1,4-\mathrm{N} \text {-acetylglucosaminyltransferase, } \\
\text { isozyme } \mathrm{C} \text { (putative) }\end{array}$ \\
\hline-2.18 & R3HDM2 & $\mathrm{R} 3 \mathrm{H}$ domain containing 2 \\
\hline-2.18 & AMDHD1 & Amidohydrolase domain containing 1 \\
\hline-2.18 & SNORA68 & Small nucleolar RNA, H/ACA box 68 \\
\hline-2.18 & SLC7A1 & Solute carrier family 7 (cationic amino acid transporter, $\mathrm{y}^{+}$system), member 1 \\
\hline-2.19 & NID2 & Nidogen 2 (osteonidogen) \\
\hline-2.19 & LXN & Latexin \\
\hline-2.19 & CTIF & CBP80/20-dependent translation initiation factor \\
\hline-2.19 & TRNAG36 & $\begin{array}{l}\text { Transfer RNA glycine } 36 \text { (anticodon CCC); transfer RNA valine } 34 \\
\text { (anticodon CAC) pseudogene }\end{array}$ \\
\hline-2.19 & ST6GAL1 & ST6 $\beta$-galactosamide $\alpha$-2,6-sialyltranferase 1 \\
\hline-2.19 & SCARNA23 & Small Cajal body-specific RNA 23 \\
\hline-2.19 & DPYD & Dihydropyrimidine dehydrogenase \\
\hline-2.19 & DDR2 & Discoidin domain receptor tyrosine kinase 2 \\
\hline-2.19 & LGI1 & Leucine-rich, glioma inactivated 1 \\
\hline-2.19 & HLA-F & Major histocompatibility complex, class I, F \\
\hline-2.20 & PSPH & Phosphoserine phosphatase \\
\hline
\end{tabular}


Table II. Continued.

\begin{tabular}{|c|c|c|}
\hline Fold-change & Gene symbol & mRNA description \\
\hline-2.20 & HBG1 & $\begin{array}{l}\text { Hemoglobin, } \gamma \mathrm{A} \text {; hemoglobin, } \gamma \mathrm{G} \text {; uncharacterized LOC100653319; } \\
\text { uncharacterized LOC100653006 }\end{array}$ \\
\hline-2.20 & TMEM231 & Transmembrane protein 231 \\
\hline-2.20 & EML2 & Echinoderm microtubule-associated protein like-2 \\
\hline-2.21 & IGF2R & Insulin-like growth factor 2 receptor \\
\hline-2.21 & PYGB & Phosphorylase, glycogen; brain \\
\hline-2.21 & WSB1 & WD repeat and SOCS box containing 1 \\
\hline-2.22 & ITGBL1 & Integrin, $\beta$-like 1 (with EGF-like repeat domains) \\
\hline-2.22 & PCBP3 & Poly(rC) binding protein 3 \\
\hline-2.22 & SERPINE2 & $\begin{array}{l}\text { Serpin peptidase inhibitor, clade E (nexin, plasminogen activator inhibitor type } 1 \text { ), } \\
\text { member } 2\end{array}$ \\
\hline-2.22 & TAPBP & TAP binding protein (tapasin) \\
\hline-2.22 & ERGIC1 & Endoplasmic reticulum-golgi intermediate compartment (ERGIC) 1 \\
\hline-2.23 & PDLIM5 & PDZ and LIM domain 5 \\
\hline-2.23 & BCAT1 & Branched chain amino-acid transaminase 1 , cytosolic \\
\hline-2.23 & RN5S198 & RNA, 5S ribosomal 198 \\
\hline-2.23 & $\mathrm{PC}$ & Pyruvate carboxylase \\
\hline-2.24 & BMPER & BMP binding endothelial regulator \\
\hline-2.24 & BACE2 & $\beta$-site APP-cleaving enzyme 2 \\
\hline-2.24 & CTNNAL1 & Catenin (cadherin-associated protein), $\alpha$-like 1 \\
\hline-2.24 & ANKRD20A11P & $\begin{array}{l}\text { Ankyrin repeat domain } 20 \text { family, member A11, pseudogene; } \\
\text { ankyrin repeat domain } 20 \text { family, member A8, pseudogene }\end{array}$ \\
\hline-2.24 & SNORD116-29 & Small nucleolar RNA, C/D box 116-29 \\
\hline-2.24 & IFRD1 & Interferon-related developmental regulator 1 \\
\hline-2.25 & EDNRB & Endothelin receptor type B \\
\hline-2.25 & VN1R4 & Vomeronasal 1 receptor 4 \\
\hline-2.26 & NR4A2 & Nuclear receptor subfamily 4 , group A, member 2 \\
\hline-2.26 & DKK1 & Dickkopf 1 homolog (Xenopus laevis) \\
\hline-2.26 & OLFML2A & Olfactomedin-like 2A \\
\hline-2.26 & C6orf48 & Chromosome 6 open reading frame 48 \\
\hline-2.27 & SPRY3 & Sprouty homolog 3 (Drosophila) \\
\hline-2.27 & PSAT1 & Phosphoserine aminotransferase 1 \\
\hline-2.27 & HOXA2 & Homeobox A2 \\
\hline-2.28 & DUSP1 & Dual specificity phosphatase 1 \\
\hline-2.28 & SLC44A1 & Solute carrier family 44 , member 1 \\
\hline-2.28 & ITPRIP & Inositol 1,4,5-trisphosphate receptor interacting protein \\
\hline-2.28 & OSMR & Oncostatin $\mathrm{M}$ receptor \\
\hline-2.28 & SUMF2 & Sulfatase modifying factor 2 \\
\hline-2.28 & S100A16 & S100 calcium binding protein A16 \\
\hline-2.28 & ITGB5 & Integrin, $\beta 5$ \\
\hline-2.28 & SLC33A1 & Solute carrier family 33 (acetyl-CoA transporter), member 1 \\
\hline-2.28 & FOSL2 & FOS-like antigen 2 \\
\hline-2.28 & RBMS2 & RNA binding motif, single-stranded interacting protein 2 \\
\hline-2.28 & SOCS2 & Suppressor of cytokine signaling 2 \\
\hline-2.29 & EIF4EBP1 & Eukaryotic translation initiation factor $4 \mathrm{E}$ binding protein 1 \\
\hline-2.29 & RN5S449 & RNA, $5 \mathrm{~S}$ ribosomal 449 \\
\hline-2.29 & IDH1 & Isocitrate dehydrogenase $1\left(\mathrm{NADP}^{+}\right)$, soluble \\
\hline-2.29 & UPP1 & Uridine phosphorylase 1 \\
\hline-2.29 & DPYSL2 & Dihydropyrimidinase-like 2 \\
\hline-2.30 & CAMK2D & Calcium/calmodulin-dependent protein kinase II $\delta$ \\
\hline-2.30 & SHMT2 & Serine hydroxymethyltransferase 2 (mitochondrial) \\
\hline-2.30 & MUC1 & Mucin 1, cell surface associated \\
\hline
\end{tabular}


Table II. Continued.

Fold-change Gene symbol mRNA description

ADAM9
LOC 100653286

CD226

ZNF449

PHLDB2

PLGRKT

SPAG4

GADD45A

CGREF1

DUSP22

PMAIP1

LOC100506948

ECE1

PAQR5

LMF1

SNORD13P2

PLEKHG2

TMX3

ZNF558

SOX9

SIL1

FSD2

SNORD116-16

ANGPTL4

PABPC1L

PLCB4

BEST1

RNU7-35P

$\mathrm{CDH} 2$

HYOU1

ITGA6

THBS3

TM4SF18

MTHFD1L

PIR

MOCOS

RHBDD1

ARL4D

F2RL1

RAPGEF5

PCMTD2

SREBF1

CPS 1

ZEB2

NRP2

HLCS
ADAM metallopeptidase domain 9

Aldo-keto reductase family 1 member C2-like; aldo-keto reductase family 1 , member $\mathrm{C} 2$ (dihydrodiol dehydrogenase 2; bile acid binding protein;

3 - $\alpha$ hydroxysteroid dehydrogenase, type III)

CD226 molecule

Zinc finger protein 449

Pleckstrin homology-like domain, family B, member 2

Plasminogen receptor, $\mathrm{C}$-terminal lysine transmembrane protein

Sperm-associated antigen 4

Growth arrest and DNA-damage-inducible, $\alpha$

Cell growth regulator with EF-hand domain 1

Dual specificity phosphatase 22; dual specificity protein phosphatase 22-like

Phorbol-12-myristate-13-acetate-induced protein 1

Uncharacterized LOC100506948; small nucleolar RNA, C/D box 116-28;

$115-26$; 115-13; 115-7; 116-22

Endothelin converting enzyme 1

Progestin and adipoQ receptor family member $\mathrm{V}$

Lipase maturation factor 1

Small nucleolar RNA, C/D box 13 pseudogene 2

Pleckstrin homology domain containing, family G (with RhoGef domain) member 2

Thioredoxin-related transmembrane protein 3

Zinc finger protein 558

SRY (sex determining region Y)-box 9

SIL1 homolog, endoplasmic reticulum chaperone (S. cerevisiae)

Fibronectin type III and SPRY domain containing 2;

small Cajal body-specific RNA 15

Small nucleolar RNA, C/D box 116-16

Angiopoietin-like 4

Poly(A) binding protein, cytoplasmic 1-like

Phospholipase C, $\beta 4$

Bestrophin 1

RNA, U7 small nuclear 35 pseudogene

Cadherin 2, type 1, N-cadherin (neuronal)

Hypoxia upregulated 1

Integrin, $\alpha 6$

Thrombospondin 3

Transmembrane $4 \mathrm{~L}$ six family member 18

Methylenetetrahydrofolate dehydrogenase (NADP ${ }^{+}$dependent) 1-like

Pirin (iron-binding nuclear protein); PIR-FIGF readthrough

Molybdenum cofactor sulfurase

Rhomboid domain containing 1

ADP-ribosylation factor-like 4D

Coagulation factor II (thrombin) receptor-like 1

Rap guanine nucleotide exchange factor (GEF) 5

Protein-L-isoaspartate (D-aspartate) $O$-methyltransferase domain containing 2; long intergenic non-protein coding RNA 266-1

Sterol regulatory element binding transcription factor 1

Carbamoyl-phosphate synthase 1 , mitochondrial

Zinc finger E-box binding homeobox 2

Neuropilin 2

Holocarboxylase synthetase [biotin-(proprionyl-CoA-carboxylase (ATP-hydrolysing) ligase] 
Table II. Continued.

\begin{tabular}{|c|c|c|}
\hline Fold-change & Gene symbol & mRNA description \\
\hline-2.40 & CRIM1 & Cysteine rich transmembrane BMP regulator 1 (chordin-like) \\
\hline-2.41 & ZC3H6 & Zinc finger $\mathrm{CCCH}$-type containing 6 \\
\hline-2.41 & ARRDC4 & Arrestin domain containing 4 \\
\hline-2.41 & PDIA5 & Protein disulfide isomerase family A, member 5 \\
\hline-2.41 & WDFY2 & WD repeat and FYVE domain containing 2 \\
\hline-2.41 & VEGFC & Vascular endothelial growth factor C \\
\hline-2.42 & CA5B & Carbonic anhydrase VB, mitochondrial \\
\hline-2.42 & CLN8 & Ceroid-lipofuscinosis, neuronal 8 (epilepsy, progressive with mental retardation) \\
\hline-2.42 & CYP20A1 & Cytochrome P450, family 20, subfamily A, polypeptide 1 \\
\hline-2.43 & AKR1B10 & Aldo-keto reductase family 1, member B10 (aldose reductase) \\
\hline-2.43 & GPR126 & G protein-coupled receptor 126 \\
\hline-2.43 & TSPAN4 & Tetraspanin 4 \\
\hline-2.43 & FGG & Fibrinogen $\gamma$ chain \\
\hline-2.44 & RORA & RAR-related orphan receptor A \\
\hline-2.44 & UGGT2 & UDP-glucose glycoprotein glucosyltransferase 2 \\
\hline-2.44 & DUSP5 & Dual specificity phosphatase 5 \\
\hline-2.45 & P4HA3 & Prolyl 4-hydroxylase, $\alpha$ polypeptide III \\
\hline-2.45 & MARS & Methionyl-tRNA synthetase \\
\hline-2.45 & IGF2BP3 & Insulin-like growth factor $2 \mathrm{mRNA}$ binding protein 3 \\
\hline-2.45 & TTC13 & Tetratricopeptide repeat domain 13 \\
\hline-2.45 & C16orf58 & Chromosome 16 open reading frame 58 \\
\hline-2.46 & BMP5 & Bone morphogenetic protein 5 \\
\hline-2.46 & SREBF2 & Sterol regulatory element binding transcription factor 2 \\
\hline-2.46 & NEDD9 & Neural precursor cell expressed, developmentally downregulated 9 \\
\hline-2.48 & OCLN & Occludin; occludin pseudogene \\
\hline-2.48 & PAG1 & Phosphoprotein associated with glycosphingolipid microdomains 1 \\
\hline-2.48 & PPFIBP1 & PTPRF interacting protein, binding protein 1 (liprin $\beta 1)$ \\
\hline-2.48 & BTG1 & B-cell translocation gene 1 , antiproliferative \\
\hline-2.48 & XYLT1 & Xylosyltransferase I \\
\hline-2.48 & CEP72 & Centrosomal protein $72 \mathrm{kDa}$ \\
\hline-2.48 & HNRNPU-AS1 & HNRNPU antisense RNA 1 (non-protein coding) \\
\hline-2.49 & CLK1 & CDC-like kinase 1 \\
\hline-2.49 & VPS13C & Vacuolar protein sorting 13 homolog C (S. cerevisiae) \\
\hline-2.50 & LAMA1 & Laminin, $\alpha 1$ \\
\hline-2.50 & PLD1 & Phospholipase D1, phosphatidylcholine-specific \\
\hline-2.50 & AGXT2L2 & Alanine-glyoxylate aminotransferase 2-like 2 \\
\hline-2.50 & SLCO5A1 & Solute carrier organic anion transporter family, member $5 \mathrm{~A} 1$ \\
\hline-2.51 & CARS & Cysteinyl-tRNA synthetase \\
\hline-2.51 & ACAD11 & $\begin{array}{l}\text { Acyl-CoA dehydrogenase family, member 11; nephronophthisis } 3 \text { (adolescent); } \\
\text { NPHP3-ACAD11 readthrough }\end{array}$ \\
\hline-2.51 & SLC2A14 & Solute carrier family 2 (facilitated glucose transporter), member 14 \\
\hline-2.52 & SCCPDH & Saccharopine dehydrogenase (putative) \\
\hline-2.52 & NT5E & 5'-nucleotidase, ecto (CD73) \\
\hline-2.53 & AADAC & Arylacetamide deacetylase (esterase) \\
\hline-2.53 & TMEM106B & Transmembrane protein 106B \\
\hline-2.54 & GDPD1 & Glycerophosphodiester phosphodiesterase domain containing 1 \\
\hline-2.55 & DNER & $\delta /$ notch-like EGF repeat containing \\
\hline-2.55 & SLITRK4 & SLIT and NTRK-like family, member 4 \\
\hline-2.55 & ST3GAL1 & ST3 $\beta$-galactoside $\alpha$-2,3-sialyltransferase 1 \\
\hline-2.55 & SLC8A1 & Solute carrier family 8 (sodium/calcium exchanger), member 1 \\
\hline-2.55 & SPDYE8P & Speedy homolog E8 (Xenopus laevis), pseudogene \\
\hline
\end{tabular}


Table II. Continued.

\begin{tabular}{|c|c|c|}
\hline Fold-change & Gene symbol & mRNA description \\
\hline-2.55 & SLC9B 1 & $\begin{array}{l}\text { Solute carrier family } 9 \text {, subfamily B (NHA1, cation proton antiporter } 1) \text {, } \\
\text { member } 1 \text {; solute carrier family } 9 \text {, subfamily B (NHA1, cation proton antiporter } 1) \text {, } \\
\text { member } 1 \text { pseudogene } 2 \text {; solute carrier family } 9 \text {, subfamily B } \\
\text { (NHA } 1 \text {, cation proton antiporter } 1) \text {, member } 1 \text { pseudogene } 3\end{array}$ \\
\hline-2.56 & ZP3 & Zona pellucida glycoprotein 3 (sperm receptor) \\
\hline-2.56 & BCAR3 & Breast cancer anti-estrogen resistance 3 \\
\hline-2.57 & GABBR2 & $\gamma$-aminobutyric acid (GABA) B receptor, 2 \\
\hline-2.57 & CD55 & CD55 molecule, decay accelerating factor for complement (Cromer blood group) \\
\hline-2.57 & GALNT3 & $\begin{array}{l}\text { UDP- } N \text {-acetyl- } \alpha \text {-D-galactosamine:polypeptide } \\
\text { N-acetylgalactosaminyltransferase } 3 \text { (GalNAc-T3) }\end{array}$ \\
\hline-2.57 & THBS1 & Thrombospondin 1 \\
\hline-2.57 & MIR21 & microRNA 21 \\
\hline-2.58 & SESN2 & Sestrin 2 \\
\hline-2.59 & FLNB & Filamin $B, \beta$ \\
\hline-2.59 & EFNA5 & Ephrin-A5 \\
\hline-2.59 & PTPRB & Protein tyrosine phosphatase, receptor type, B \\
\hline-2.59 & PION & Pigeon homolog (Drosophila) \\
\hline-2.60 & EFEMP1 & EGF containing fibulin-like extracellular matrix protein 1 \\
\hline-2.60 & DUSP4 & Dual specificity phosphatase 4 \\
\hline-2.61 & RTN1 & Reticulon 1 \\
\hline-2.61 & DGKD & Diacylglycerol kinase, $\delta 130 \mathrm{kDa}$ \\
\hline-2.61 & ANTXR1 & Anthrax toxin receptor 1 \\
\hline-2.61 & SUCLG2 & Succinate-CoA ligase, GDP-forming, $\beta$ subunit \\
\hline-2.61 & LOC 100506965 & Uncharacterized LOC100506965 \\
\hline-2.62 & LAMC1 & laminin, $\gamma 1$ (formerly LAMB2) \\
\hline-2.63 & CEP70 & Centrosomal protein $70 \mathrm{kDa}$ \\
\hline-2.64 & ABCA8 & ATP-binding cassette, sub-family A (ABC1), member 8 \\
\hline-2.65 & SERPINB9 & Serpin peptidase inhibitor, clade B (ovalbumin), member 9 \\
\hline-2.65 & PDE1C & Phosphodiesterase 1C, calmodulin-dependent 70 kDa \\
\hline-2.65 & ACSS3 & Acyl-CoA synthetase short-chain family member 3 \\
\hline-2.66 & SCG2 & Secretogranin II \\
\hline-2.67 & SLC39A8 & Solute carrier family 39 (zinc transporter), member 8 \\
\hline-2.67 & $\mathrm{~F} 2 \mathrm{R}$ & Coagulation factor II (thrombin) receptor \\
\hline-2.67 & MME & Membrane metallo-endopeptidase \\
\hline-2.68 & LCP1 & Lymphocyte cytosolic protein 1 (L-plastin) \\
\hline-2.68 & $\mathrm{CFH}$ & Complement factor $\mathrm{H}$ \\
\hline-2.68 & NCAM2 & Neural cell adhesion molecule 2 \\
\hline-2.70 & HINT3 & Histidine triad nucleotide binding protein 3 \\
\hline-2.70 & GPR37 & G protein-coupled receptor 37 (endothelin receptor type B-like) \\
\hline-2.71 & PPAP2B & Phosphatidic acid phosphatase type 2B \\
\hline-2.72 & LAMB1 & Laminin, $\beta 1$ \\
\hline-2.73 & GALNTL1 & $\begin{array}{l}\text { UDP- } N \text {-acetyl- } \alpha \text {-D-galactosamine:polypeptide } \\
\mathrm{N} \text {-acetylgalactosaminyltransferase-like } 1\end{array}$ \\
\hline-2.73 & PDK1 & Pyruvate dehydrogenase kinase, isozyme 1 \\
\hline-2.74 & TRMT2B & tRNA methyltransferase 2 homolog B (S. cerevisiae) \\
\hline-2.74 & SSFA2 & Sperm-specific antigen 2 \\
\hline-2.75 & TMEM100 & Transmembrane protein 100 \\
\hline-2.75 & RAB39B & RAB39B, member RAS oncogene family \\
\hline-2.75 & SORBS2 & Sorbin and SH3 domain containing 2 \\
\hline-2.76 & RGS2 & Regulator of G-protein signaling 2,24 kDa \\
\hline-2.76 & SERPIND1 & Serpin peptidase inhibitor, clade D (heparin cofactor), member 1 \\
\hline-2.77 & LOC344887 & NmrA-like family domain containing 1 pseudogene \\
\hline
\end{tabular}


Table II. Continued.

\begin{tabular}{|c|c|c|}
\hline Fold-change & Gene symbol & mRNA description \\
\hline-2.77 & PRKAA2 & Protein kinase, AMP-activated, $\alpha 2$ catalytic subunit \\
\hline-2.77 & PYCR1 & Pyrroline-5-carboxylate reductase 1 \\
\hline-2.77 & MIR32 & microRNA 32 \\
\hline-2.77 & TTC32 & Tetratricopeptide repeat domain 32 \\
\hline-2.78 & CCDC68 & Coiled-coil domain containing 68 \\
\hline-2.78 & GPR22 & G protein-coupled receptor 22 \\
\hline-2.79 & ULBP1 & UL16 binding protein 1 \\
\hline-2.79 & AXL & AXL receptor tyrosine kinase \\
\hline-2.79 & ERVK-9 & $\begin{array}{l}\text { Endogenous retrovirus group } \mathrm{K} \text {, member } 9 \text {; endogenous retrovirus group } \mathrm{K} \text {, } \\
\text { member } 4 \text {; endogenous retrovirus group } \mathrm{K} \text {, member } 19\end{array}$ \\
\hline-2.79 & DOCK4 & Dedicator of cytokinesis 4 \\
\hline-2.80 & PTPRM & Protein tyrosine phosphatase, receptor type, $\mathrm{M}$ \\
\hline-2.80 & STC2 & Stanniocalcin 2 \\
\hline-2.80 & PHLDA1 & Pleckstrin homology-like domain, family A, member 1 \\
\hline-2.81 & FAM198B & Family with sequence similarity 198, member B \\
\hline-2.81 & NR1D1 & Nuclear receptor subfamily 1 , group D, member 1 \\
\hline-2.82 & NUP210 & Nucleoporin $210 \mathrm{kDa}$ \\
\hline-2.83 & PCID2 & PCI domain containing 2 \\
\hline-2.83 & ZBTB38 & Zinc finger and BTB domain containing 38 \\
\hline-2.83 & PIGF & Phosphatidylinositol glycan anchor biosynthesis, class F \\
\hline-2.83 & GLRXP3 & Glutaredoxin (thioltransferase) pseudogene 3 \\
\hline-2.74 & SSFA2 & Sperm specific antigen 2 \\
\hline-2.84 & HSPA13 & Heat shock protein $70 \mathrm{kDa}$ family, member 13 \\
\hline-2.85 & RBMS3 & RNA binding motif, single stranded interacting protein 3 \\
\hline-2.88 & SMYD3 & SET and MYND domain containing 3 \\
\hline-2.88 & ASNS & Asparagine synthetase (glutamine-hydrolyzing) \\
\hline-2.89 & TFPI2 & Tissue factor pathway inhibitor 2 \\
\hline-2.89 & TNFRSF19 & Tumor necrosis factor receptor superfamily, member 19 \\
\hline-2.90 & CRY1 & Cryptochrome 1 (photolyase-like) \\
\hline-2.90 & IL1RAP & Interleukin 1 receptor accessory protein \\
\hline-2.91 & AGA & Aspartyl glucose aminidase \\
\hline-2.93 & FBXO32 & F-box protein 32 \\
\hline-2.94 & LIF & Leukemia inhibitory factor \\
\hline-2.94 & TMEM194B & Transmembrane protein 194B \\
\hline-2.95 & FAM115A & Family with sequence similarity 115 , member A \\
\hline-2.96 & PTPRD & Protein tyrosine phosphatase, receptor type, D \\
\hline-2.98 & HERPUD1 & $\begin{array}{l}\text { Homocysteine-inducible, endoplasmic reticulum stress-inducible, } \\
\text { ubiquitin-like domain member } 1\end{array}$ \\
\hline-2.98 & ALPK2 & $\alpha$-kinase 2 \\
\hline-3.00 & BMP6 & Bone morphogenetic protein 6 \\
\hline-3.00 & LONP1 & Lon peptidase 1 , mitochondrial \\
\hline-3.02 & CCPG1 & Cell cycle progression 1 ; DYX1C1-CCPG1 readthrough (non-protein coding) \\
\hline-3.03 & CHAC1 & ChaC, cation transport regulator homolog 1 (E. coli) \\
\hline-3.04 & XBP1 & $\mathrm{X}$-box binding protein 1 \\
\hline-3.05 & FHAD1 & Forkhead-associated (FHA) phosphopeptide binding domain 1 \\
\hline-3.07 & GREM2 & Gremlin 2 \\
\hline-3.07 & CXCL2 & Chemokine (C-X-C motif) ligand 2 \\
\hline-3.07 & KLRC3 & Killer cell lectin-like receptor subfamily $\mathrm{C}$, member 3; member 2 \\
\hline-3.08 & CD36 & CD36 molecule (thrombospondin receptor) \\
\hline-3.08 & LINC00173 & Long intergenic non-protein coding RNA 173 \\
\hline-3.08 & MIRLET7F1 & microRNA let-7f-1 \\
\hline-3.09 & GDF15 & Growth differentiation factor 15 \\
\hline
\end{tabular}


Table II. Continued.

\begin{tabular}{|c|c|c|}
\hline Fold-change & Gene symbol & mRNA description \\
\hline-3.09 & VEGFA & Vascular endothelial growth factor A \\
\hline-3.11 & COL4A6 & Collagen, type IV, $\alpha 6$ \\
\hline-3.11 & ANTXR2 & Anthrax toxin receptor 2 \\
\hline-3.12 & EDIL3 & EGF-like repeats and discoidin I-like domains 3 \\
\hline-3.12 & SEPP1 & Selenoprotein P, plasma, 1 \\
\hline-3.12 & COL4A5 & Collagen, type IV, $\alpha 5$ \\
\hline-3.12 & RNF19A & Ring finger protein 19A, E3 ubiquitin protein ligase \\
\hline-3.12 & SNRPA1 & Small nuclear ribonucleoprotein polypeptide $\mathrm{A}^{\prime}$ \\
\hline-3.12 & P4HA1 & Prolyl 4-hydroxylase, $\alpha$ polypeptide I \\
\hline-3.13 & ANK2 & Ankyrin 2, neuronal \\
\hline-3.13 & TUBE1 & Tubulin, $\varepsilon 1$ \\
\hline-3.14 & GPR65 & G protein-coupled receptor 65 \\
\hline-3.15 & FRK & Fyn-related kinase \\
\hline-3.15 & SLC2A13 & Solute carrier family 2 (facilitated glucose transporter), member 13 \\
\hline-3.15 & $\mathrm{CTH}$ & Cystathionase (cystathionine $\gamma$-lyase) \\
\hline-3.15 & PLA2G7 & Phospholipase A2, group VII (platelet-activating factor acetylhydrolase, plasma) \\
\hline-3.16 & PCK2 & Phosphoenolpyruvate carboxykinase 2 (mitochondrial) \\
\hline-3.17 & PRSS23 & Protease, serine, 23 \\
\hline-3.18 & FBXL13 & F-box and leucine-rich repeat protein 13 \\
\hline-3.18 & B4GALT1 & UDP-Gal: $\beta$ GlcNAc $\beta$ 1,4-galactosyltransferase, polypeptide 1 \\
\hline-3.19 & CXCL5 & Chemokine (C-X-C motif) ligand 5 \\
\hline-3.19 & PHGDH & Phosphoglycerate dehydrogenase \\
\hline-3.19 & SLC4A7 & Solute carrier family 4, sodium bicarbonate cotransporter, member 7 \\
\hline-3.19 & DDIT4 & DNA-damage-inducible transcript 4 \\
\hline-3.21 & RSPO3 & R-spondin 3 \\
\hline-3.21 & OGT & O-linked N-acetylglucosamine (GlcNAc) transferase \\
\hline-3.22 & FAM129A & Family with sequence similarity 129, member A \\
\hline-3.22 & CDH13 & Cadherin $13, \mathrm{H}$-cadherin (heart) \\
\hline-3.23 & PLAU & Plasminogen activator, urokinase \\
\hline-3.24 & SEMA3C & $\begin{array}{l}\text { Sema domain, immunoglobulin domain (Ig), short basic domain, } \\
\text { secreted, (semaphorin) 3C }\end{array}$ \\
\hline-3.26 & CTBS & Chitobiase, di-N-acetyl- \\
\hline-3.27 & SLC4A11 & Solute carrier family 4, sodium borate transporter, member 11 \\
\hline-3.28 & CSGALNACT1 & Chondroitin sulfate $\mathrm{N}$-acetylgalactosaminyltransferase 1 \\
\hline-3.29 & PDE1A & Phosphodiesterase 1A, calmodulin-dependent \\
\hline-3.29 & BDNF & Brain-derived neurotrophic factor \\
\hline-3.29 & SAMD13 & Sterile $\alpha$ motif domain containing 13 \\
\hline-3.30 & KLRC4-KLRK1 & $\begin{array}{l}\text { KLRC4-KLRK1 readthrough; killer cell lectin-like receptor subfamily K, } \\
\text { member } 1\end{array}$ \\
\hline-3.31 & NRN1 & Neuritin 1 \\
\hline-3.31 & SEL1L3 & Sel-1 suppressor of lin-12-like 3 (C.elegans) \\
\hline-3.33 & PDE3A & Phosphodiesterase 3A, cGMP-inhibited \\
\hline-3.33 & FBN2 & Fibrillin 2 \\
\hline-3.33 & NCAM1 & Neural cell adhesion molecule 1 \\
\hline-3.34 & RAB31 & RAB31, member RAS oncogene family \\
\hline-3.35 & DEFB1 & Defensin, $\beta 1$ \\
\hline-3.36 & PLAUR & Plasminogen activator, urokinase receptor \\
\hline-3.36 & TSC22D3 & TSC22 domain family, member 3 \\
\hline-3.36 & CNTN1 & Contactin 1 \\
\hline-3.37 & PCSK1 & Proprotein convertase subtilisin/kexin type 1 \\
\hline-3.38 & ADAMTS6 & ADAM metallopeptidase with thrombospondin type 1 motif, 6 \\
\hline-3.40 & LOXL2 & Lysyl oxidase-like 2 \\
\hline
\end{tabular}


Table II. Continued.

\begin{tabular}{|c|c|c|}
\hline Fold-change & Gene symbol & mRNA description \\
\hline-3.40 & LURAP1L & Leucine rich adaptor protein 1-like \\
\hline-3.41 & $\mathrm{SCD}$ & Stearoyl-CoA desaturase ( $\delta$-9-desaturase) \\
\hline-3.42 & PSD3 & Pleckstrin and Sec7 domain containing 3 \\
\hline-3.43 & EPSTI1 & Epithelial stromal interaction 1 (breast) \\
\hline-3.44 & PLA2R1 & Phospholipase A2 receptor $1,180 \mathrm{kDa}$ \\
\hline-3.45 & CDR1 & Cerebellar degeneration-related protein $1,34 \mathrm{kDa}$ \\
\hline-3.46 & CYR61 & Cysteine-rich, angiogenic inducer, 61 \\
\hline-3.46 & KLRC2 & Killer cell lectin-like receptor subfamily $\mathrm{C}$, member 2 \\
\hline-3.46 & BHLHE40 & Basic helix-loop-helix family, member e40 \\
\hline-3.47 & FSTL1 & Follistatin-like 1 \\
\hline-3.52 & SLC7A2 & Solute carrier family 7 (cationic amino acid transporter, $\mathrm{y}^{+}$system), member 2 \\
\hline-3.53 & GTPBP2 & GTP binding protein 2 \\
\hline-3.53 & THSD4 & Thrombospondin, type I, domain containing 4 \\
\hline-3.54 & CPED1 & Cadherin-like and PC-esterase domain containing 1 \\
\hline-3.54 & ESM1 & Endothelial cell-specific molecule 1 \\
\hline-3.54 & SEMA3A & $\begin{array}{l}\text { Sema domain, immunoglobulin domain (Ig), short basic domain, } \\
\text { secreted, (semaphorin) 3A }\end{array}$ \\
\hline-3.55 & ASS1P11 & Argininosuccinate synthetase 1 pseudogene 11 \\
\hline-3.56 & ITGA3 & Integrin, $\alpha 3$ (antigen CD49C, $\alpha 3$ subunit of VLA-3 receptor) \\
\hline-3.57 & SAT1 & Spermidine/spermine N1-acetyltransferase 1 \\
\hline-3.57 & PYROXD1 & Pyridine nucleotide-disulphide oxidoreductase domain 1 \\
\hline-3.59 & WDR69 & WD repeat domain 69 \\
\hline-3.59 & SLC6A9 & Solute carrier family 6 (neurotransmitter transporter, glycine), member 9 \\
\hline-3.62 & PAPPA2 & Pappalysin 2 \\
\hline-3.63 & MALAT1 & Metastasis-associated lung adenocarcinoma transcript 1 (non-protein coding) \\
\hline-3.64 & LAMA5 & Laminin, $\alpha 5$ \\
\hline-3.65 & EDN1 & Endothelin 1 \\
\hline-3.71 & SMOX & Spermine oxidase \\
\hline-3.72 & MIR29A & microRNA 29a \\
\hline-3.74 & TFPI & Tissue factor pathway inhibitor (lipoprotein-associated coagulation inhibitor) \\
\hline-3.75 & GLRX & Glutaredoxin (thioltransferase) \\
\hline-3.79 & GPRIN3 & GPRIN family member 3 \\
\hline-3.79 & TRPC6 & Transient receptor potential cation channel, subfamily C, member 6 \\
\hline-3.81 & IL8 & Interleukin 8 \\
\hline-3.86 & IL18R1 & Interleukin 18 receptor 1 \\
\hline-3.89 & LAMB3 & Laminin, $\beta 3$ \\
\hline-3.90 & PDCD1LG2 & Programmed cell death 1 ligand 2 \\
\hline-3.93 & STARD4 & StAR-related lipid transfer (START) domain containing 4 \\
\hline-3.93 & DLG2 & Discs, large homolog 2 (Drosophila) \\
\hline-3.96 & LSAMP & Limbic system-associated membrane protein \\
\hline-4.00 & PITPNC1 & Phosphatidylinositol transfer protein, cytoplasmic 1 \\
\hline-4.03 & APLN & Apelin \\
\hline-4.03 & CTGF & Connective tissue growth factor \\
\hline-4.06 & LRRC63 & Leucine rich repeat containing 63 \\
\hline-4.08 & PLAT & Plasminogen activator, tissue \\
\hline-4.15 & SLC25A37 & Solute carrier family 25 (mitochondrial iron transporter), member 37 \\
\hline-4.19 & NUPR1 & Nuclear protein, transcriptional regulator, 1 \\
\hline-4.23 & TSPAN8 & Tetraspanin 8 \\
\hline-4.24 & SPOCK1 & Sparc/osteonectin, cwcv and kazal-like domains proteoglycan (testican) 1 \\
\hline-4.25 & NRCAM & Neuronal cell adhesion molecule \\
\hline-4.25 & C3orf49 & Chromosome 3 open reading frame 49 \\
\hline-4.35 & TMEM144 & Transmembrane protein 144 \\
\hline
\end{tabular}


Table II. Continued.

\begin{tabular}{|c|c|c|}
\hline Fold-change & Gene symbol & mRNA description \\
\hline-4.41 & PARK2 & Parkinson protein 2, E3 ubiquitin protein ligase (parkin) \\
\hline-4.44 & SCARA5 & Scavenger receptor class A, member 5 (putative) \\
\hline-4.53 & ABCC3 & ATP-binding cassette, sub-family C (CFTR/MRP), member 3 \\
\hline-4.60 & THSD7A & Thrombospondin, type I, domain containing 7A \\
\hline-4.66 & IL6 & Interleukin 6 (interferon, $\beta 2$ ) \\
\hline-4.67 & CDH19 & Cadherin 19, type 2 \\
\hline-4.70 & VLDLR & Very low density lipoprotein receptor \\
\hline-4.78 & STC1 & Stanniocalcin 1 \\
\hline-4.81 & ABI3BP & ABI family, member 3 (NESH) binding protein \\
\hline-4.90 & TNFAIP3 & Tumor necrosis factor, $\alpha$-induced protein 3 \\
\hline-4.90 & DUSP6 & Dual specificity phosphatase 6 \\
\hline-4.90 & SEMA3D & $\begin{array}{l}\text { Sema domain, immunoglobulin domain (Ig), short basic domain, } \\
\text { secreted, (semaphorin) 3D }\end{array}$ \\
\hline-4.93 & CBS & Cystathionine- $\beta$-synthase \\
\hline-4.95 & DPP10 & Dipeptidyl-peptidase 10 (non-functional) \\
\hline-5.19 & SLC16A4 & Solute carrier family 16 , member 4 (monocarboxylic acid transporter 5) \\
\hline-5.22 & CLDN1 & Claudin 1 \\
\hline-5.28 & KIAA0825 & KIAA0825 \\
\hline-5.35 & PLAC4 & Placenta-specific 4 \\
\hline-5.38 & EPHA4 & EPH receptor A4 \\
\hline-5.48 & ALDH1L2 & Aldehyde dehydrogenase 1 family, member L2 \\
\hline-5.51 & DDIT3 & DNA-damage-inducible transcript 3 \\
\hline-5.51 & TXNIP & Thioredoxin interacting protein \\
\hline-5.52 & WARS & Tryptophanyl-tRNA synthetase \\
\hline-5.69 & PRICKLE1 & Prickle homolog 1 (Drosophila) \\
\hline-5.81 & CLGN & Calmegin \\
\hline-5.81 & ERRFI1 & ERBB receptor feedback inhibitor 1 \\
\hline-5.94 & SMOC1 & SPARC related modular calcium binding 1 \\
\hline-6.03 & ABCC9 & ATP-binding cassette, sub-family C (CFTR/MRP), member 9 \\
\hline-6.05 & ASS1 & Argininosuccinate synthase 1 \\
\hline-6.20 & KCNE4 & Potassium voltage-gated channel, Isk-related family, member 4 \\
\hline-6.23 & C12orf39 & Chromosome 12 open reading frame 39 \\
\hline-6.24 & LCN1 & Lipocalin 1 \\
\hline-6.28 & PAPPA & Pregnancy-associated plasma protein A, pappalysin 1 \\
\hline-6.44 & TMEM27 & Transmembrane protein 27 \\
\hline-6.70 & CHL1 & Cell adhesion molecule with homology to L1CAM (close homolog of L1) \\
\hline-6.83 & SUCNR1 & Succinate receptor 1 \\
\hline-7.16 & AREG & Amphiregulin \\
\hline-7.57 & HPGDS & Hematopoietic prostaglandin D synthase \\
\hline-7.62 & ANXA3 & Annexin A3 \\
\hline-8.16 & SLC2A3 & Solute carrier family 2 (facilitated glucose transporter), member 3 \\
\hline-8.34 & TGFB2 & Transforming growth factor, $\beta 2$ \\
\hline-8.40 & GABRE & $\gamma$-aminobutyric acid (GABA) A receptor, $\varepsilon$; microRNA 452; microRNA 224 \\
\hline-8.88 & RNY1P5 & RNA, Ro-associated Y1 pseudogene 5 \\
\hline-9.52 & RPE65 & Retinal pigment epithelium-specific protein $65 \mathrm{kDa}$ \\
\hline-9.54 & CD274 & CD274 molecule \\
\hline-12.33 & FST & Follistatin \\
\hline-12.69 & GPR133 & G protein-coupled receptor 133 \\
\hline-13.11 & SERPINB11 & Serpin peptidase inhibitor, clade B (ovalbumin), member 11 (gene/pseudogene) \\
\hline-15.73 & IL1RL1 & Interleukin 1 receptor-like 1 \\
\hline
\end{tabular}




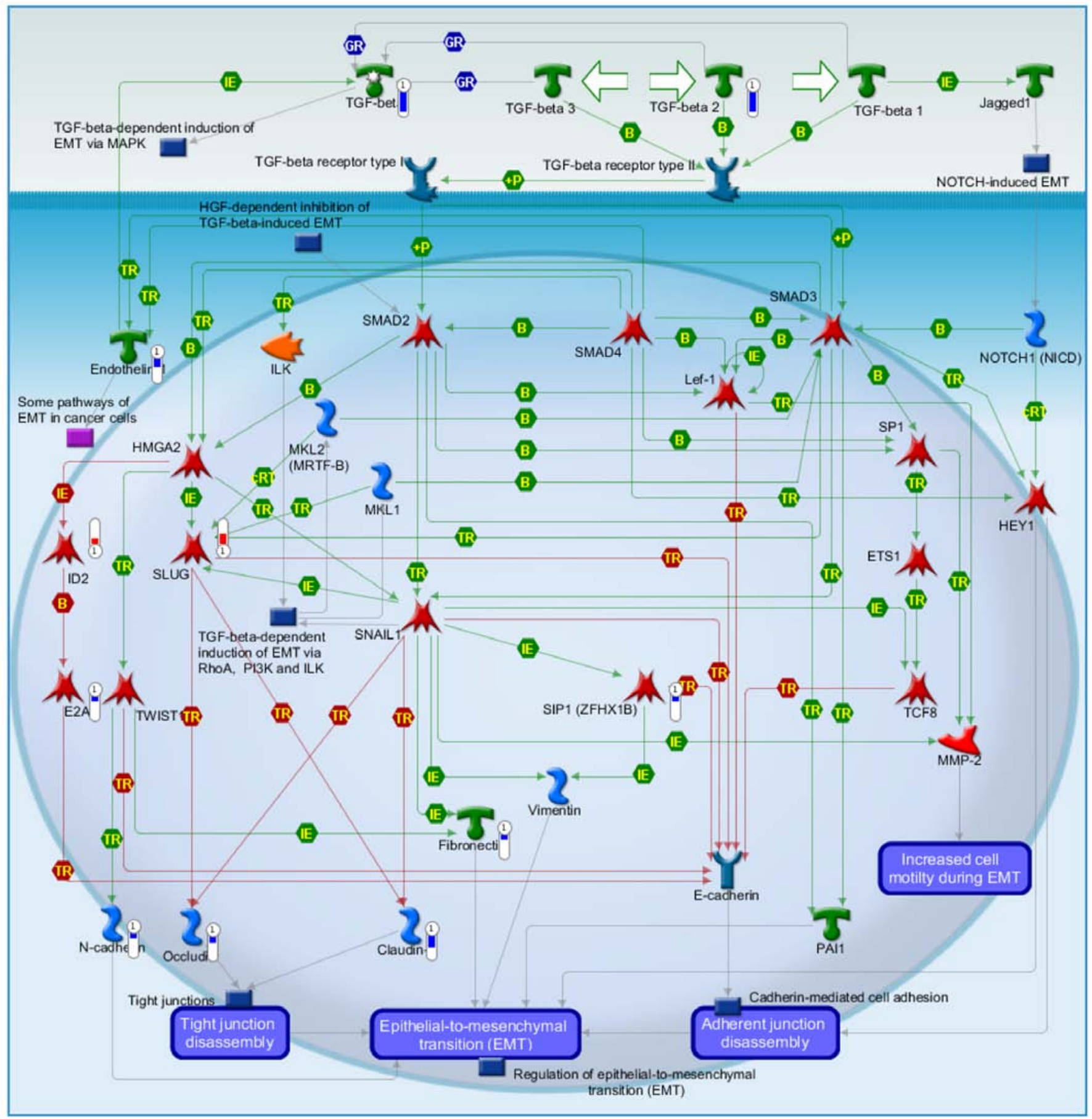

Figure 1. The top scored map (map with the lowest p-value) based on the enrichment distribution sorted by the 'Statistically significant Maps' set. Experimental data from all the files are linked to and visualized on the maps as thermometer-like figures. Upward thermometers (red) indicate upregulated signals and downward (blue) ones indicate downregulated gene expression levels.

The results also show that curcumin decreased several gene expressions associated with DNA damage, cell cycle, cell survival and cell migration and invasion. For example, DDIT3, DDIT4, GADD45A and CGREF1 were decreased 5.51-, 3.19-, 2.31- and 2.31-fold, respectively, which was associated with DNA damage. Curcumin decreased 3.02-fold of the CCPG1 gene expression which was associated with cell cycle progression and the TNFRSF10B, TSSC1, GAS5 and TNFRSF11B genes were decreased 2.17-, 2.03-, 2.03- and 2.02-fold, which was associated with cell survival. Furthermore, curcumin decreased two gene expressions at 2.15- and 2.06-fold, respectively, which associated with cell migration. It was reported that the induction of DNA-damage-inducible transcript 3 (DDIT3) was observed in the liver of mice exposed to coal mining and this gene is frequently upregulated in response to cell stress and DNA damage (33). DNA-damage-inducible transcript 4 (DDIT4) is involved in multiple biological settings such as inhibiting mTORC1 signaling and regulating the production of reactive oxygen species $(35,36)$, which was also associated with DNA damage. It was demonstrated that 


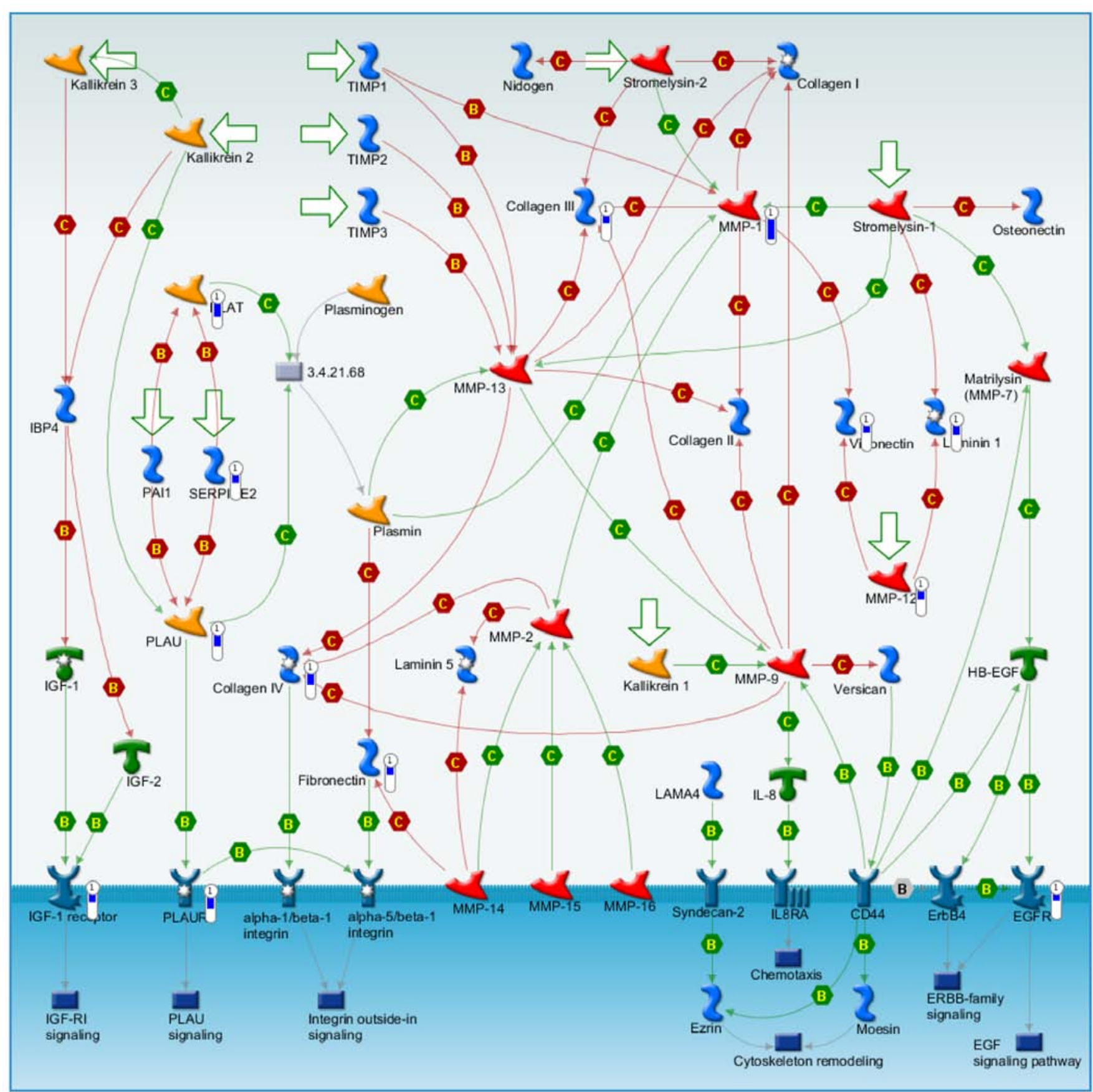

Figure 2. The second scored map (map with the second lowest p-value) based on the enrichment distribution sorted by the 'Statistically significant Maps' set. Experimental data from all the files are linked to and visualized on the maps as thermometer-like images. Upward thermometers (red) and indicate upregulated signals and downward (blue) ones indicate downregulated gene expression levels.

myristicin, an allylbenzene, is a major active component of various spices inducing DNA damage signalling (ATM) and stress response (GADD45A and GADD45G) in leukemia cells (34). The TNFRSF10B gene which encodes the apoptotic death receptor TNFRSF10B was associated with cell death (38). It was also reported that the recurrent amplification of MYC and TNFRSF11B in 8q24 is associated with poor survival in patients with gastric cancer survival (35). Growth arrest-specific 5 (GAS5) is a non-coding gene that hosts a number of small nucleolar RNAs (snoRNAs) and it has been suggested to play a tumor suppressive role $(36,37)$.
The results of the present study indicated that a number of genes were associated with DNA damage and repair, cell cycle check point, cell survival and cell migration and invasion in NCI-H460 cells following exposure to curcumin. Based on these observations, we determined these associated genes with possible signaling complex interactions. Thus, future investigations are needed to expand or append our current findings and possible understandings. These genes were affected by curcumin, which provides more information for the understanding of the cytotoxic mechanism of curcumin at the genetic level. Furthermore, it provides potentially useful 


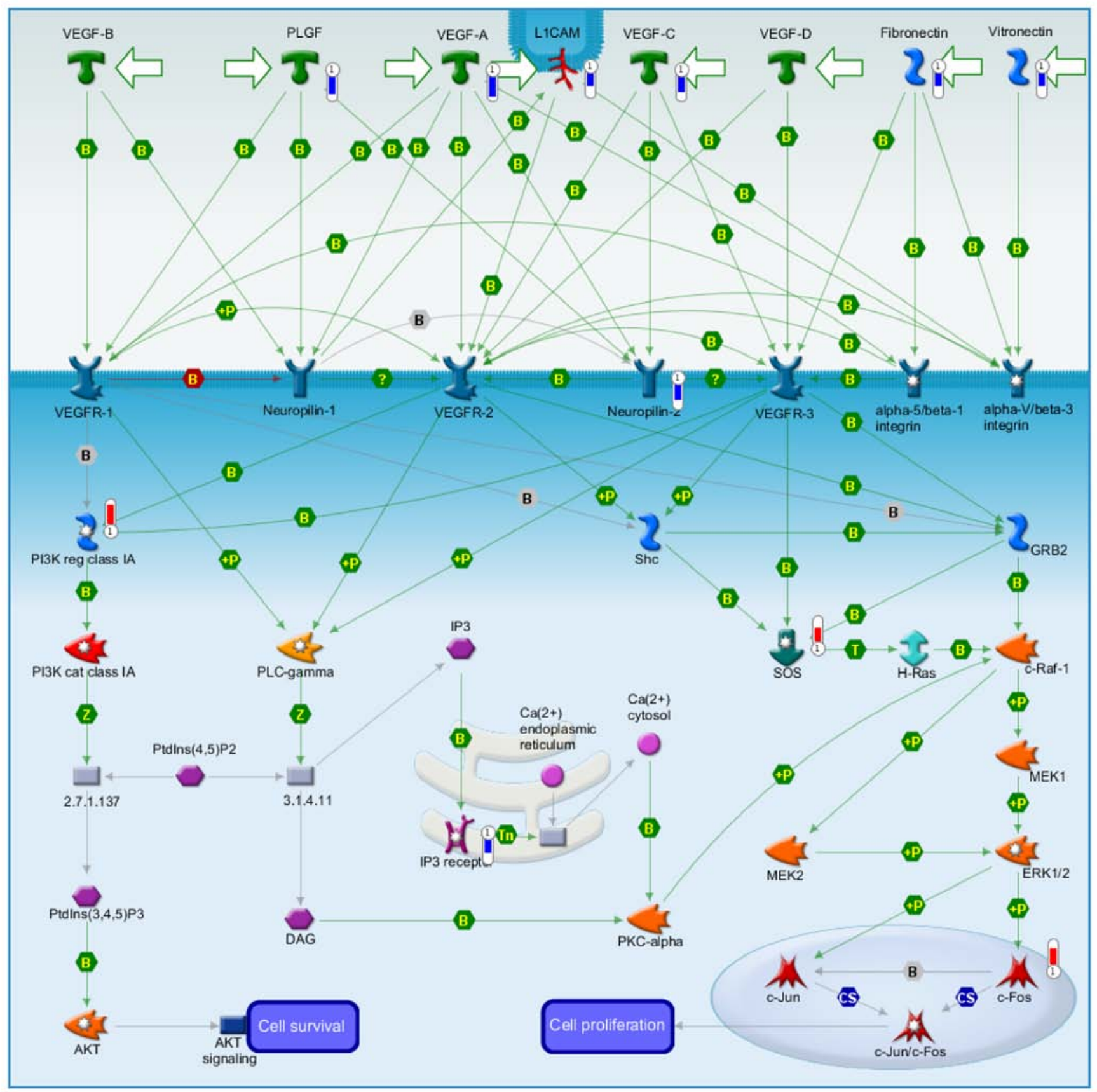

Figure 3. The third scored map (map with the third lowest p-value) based on the enrichment distribution sorted by the 'Statistically significant Maps' set. Experimental data from all the files are linked to and visualized on the maps as thermometer-like figures. Upward thermometers (red) indicate upregulated signals and downward (blue) ones indicate downregulated gene expression levels.

biomarkers or targets in clinic for the diagnosis and treatment of human lung cancer.

\section{Acknowledgements}

This study was supported by grant RD2015-032 from the National Yang-Ming University Hospital, Yilan, Taiwan.

\section{References}

1. Buyukcelik A, Yalcin B and Utkan G: Multidisciplinary management of lung cancer. N Engl J Med 350: 2008-2010, 2004.
2. Jemal A, Bray F, Center MM, Ferlay J, Ward E and Forman D: Global cancer statistics. CA Cancer J Clin 61: 69-90, 2011.

3. McCulloch M, See C, Shu XJ, Broffman M, Kramer A, Fan WY, Gao J, Lieb W, Shieh K and Colford JM Jr: Astragalus-based Chinese herbs and platinum-based chemotherapy for advanced non-small-cell lung cancer: Meta-analysis of randomized trials. J Clin Oncol 24: 419-430, 2006.

4. Tsai MF, Wang CC and Chen JJ: Tumour suppressor HLJ1: A potential diagnostic, preventive and therapeutic target in non-small cell lung cancer. World J Clin Oncol 5: 865-873, 2014.

5. Prasad S, Tyagi AK and Aggarwal BB: Recent developments in delivery, bioavailability, absorption and metabolism of curcumin: The golden pigment from golden spice. Cancer Res Treat 46: 2-18, 2014.

6. Hasima N and Aggarwal BB: Cancer-linked targets modulated by curcumin. Int J Biochem Mol Biol 3: 328-351, 2012. 
7. Campbell FC and Collett GP: Chemopreventive properties of curcumin. Future Oncol 1: 405-414, 2005.

8. Sarkar FH and Li Y: Cell signaling pathways altered by natural chemopreventive agents. Mutat Res 555: 53-64, 2004.

9. Shishodia S, Sethi G and Aggarwal BB: Curcumin: Getting back to the roots. Ann NY Acad Sci 1056: 206-217, 2005.

10. Zanotto-Filho A, Braganhol E, Edelweiss MI, Behr GA, Zanin R, Schröder R, Simões-Pires A, Battastini AM and Moreira JC: The curry spice curcumin selectively inhibits cancer cells growth in vitro and in preclinical model of glioblastoma. J Nutr Biochem 23: 591-601, 2012.

11. Xiao K, Jiang J, Guan C, Dong C, Wang G, Bai L, Sun J, Hu C and Bai C: Curcumin induces autophagy via activating the AMPK signaling pathway in lung adenocarcinoma cells. J Pharmacol Sci 123: 102-109, 2013.

12. Chen QY, Zheng Y, Jiao DM, Chen FY, Hu HZ, Wu YQ, Song J, Yan J, Wu LJ and Lv GY: Curcumin inhibits lung cancer cell migration and invasion through Rac1-dependent signaling pathway. J Nutr Biochem 25: 177-185, 2014.

13. Lev-Ari S, Starr A, Katzburg S, Berkovich L, Rimmon A Ben-Yosef R, Vexler A, Ron I and Earon G: Curcumin induces apoptosis and inhibits growth of orthotopic human non-small cell lung cancer xenografts. J Nutr Biochem 25: 843-850, 2014.

14. Shen Y, Zhang S, Huang X, Chen K, Shen J and Wang Z: Involvement of p53 mutation and mismatch repair proteins dysregulation in NNK-induced malignant transformation of human bronchial epithelial cells. Biomed Res Int 2014: 920275 , 2014.

15. Muller PA and Vousden KH: p53 mutations in cancer. Nat Cell Biol 15: 2-8, 2013.

16. Bunn PA Jr: Worldwide overview of the current status of lung cancer diagnosis and treatment. Arch Pathol Lab Med 136 1478-1481, 2012.

17. Eberhard DA, Johnson BE, Amler LC, Goddard AD, Heldens SL, Herbst RS, Ince WL, Jänne PA, Januario T, Johnson DH, et al: Mutations in the epidermal growth factor receptor and in KRAS are predictive and prognostic indicators in patients with nonsmall-cell lung cancer treated with chemotherapy alone and in combination with erlotinib. J Clin Oncol 23: 5900-5909, 2005

18. Kwak EL, Bang YJ, Camidge DR, Shaw AT, Solomon B Maki RG, Ou SH, Dezube BJ, Jänne PA, Costa DB, et al: Anaplastic lymphoma kinase inhibition in non-small-cell lung cancer. N Engl J Med 363: 1693-1703, 2010.

19. Wu SH, Hsiao YT, Chen JC, Lin JH, Hsu SC, Hsia TC, Yang ST, Hsu WH and Chung JG: Bufalin alters gene expressions associated DNA damage, cell cycle, and apoptosis in human lung cancer NCI-H460 cells in vitro. Molecules 19: 6047-6057, 2014.

20. Gardina PJ, Clark TA, Shimada B, Staples MK, Yang Q, Veitch J, Schweitzer A, Awad T, Sugnet C, Dee S, et al: Alternative splicing and differential gene expression in colon cancer detected by a whole genome exon array. BMC Genomics 7: 325, 2006.

21. Li Z, Meng Q, Yu Q, Zhou Z and Li L: Evaluation of c-myc and CCNE2 amplification in breast cancer with quantitative multigene fluorescence in-situ hybridization. Zhonghua Bing Li Xue Za Zhi 43: 455-458, 2014 (In Chinese).

22. Kawase T, Ichikawa H, Ohta T, Nozaki N, Tashiro F, Ohki R and Taya Y: p53 target gene $A E N$ is a nuclear exonuclease required for p53-dependent apoptosis. Oncogene 27: 3797-3810, 2008
23. Shahbazi J, Lock R and Liu T: Tumor protein 53-induced nuclear protein 1 enhances 53 function and represses tumorigenesis. Front Genet 4: 80, 2013

24. Kim GS, Kang J, Bang SW and Hwang DS: Cdc6 localizes to $\mathrm{S}$ - and G2-phase centrosomes in a cell cycle-dependent manner. Biochem Biophys Res Commun 456: 763-767, 2015.

25. Bidkhori G, Narimani Z, Hosseini Ashtiani S, Moeini A, Nowzari-Dalini A and Masoudi-Nejad A: Reconstruction of an integrated genome-scale co-expression network reveals key modules involved in lung adenocarcinoma. PLoS One 8: e67552, 2013.

26. Davidson G and Niehrs C: Emerging links between CDK cell cycle regulators and Wnt signaling. Trends Cell Biol 20: 453-460, 2010.

27. Ding Y, Zhu W, Sun R, Yuan G, Zhang D, Fan Y and Sun J: Diphenylene iodonium interferes with cell cycle progression and induces apoptosis by modulating NAD(P)H oxidase/ROS/cell cycle regulatory pathways in Burkitt's lymphoma cells. Oncol Rep 33: 1434-1442, 2015.

28. Jiang DS, Wei X, Zhang XF, Liu Y, Zhang Y, Chen K, Gao L, Zhou H, Zhu XH, Liu PP, et al: IRF8 suppresses pathological cardiac remodelling by inhibiting calcineurin signalling. Nat Commun 5: 3303, 2014

29. Li L, Chen W, Zhu Y, Wang X, Jiang DS, Huang F, Wang L, Xiang F, Qin W, Wang Q, et al: Caspase recruitment domain 6 protects against cardiac hypertrophy in response to pressure overload. Hypertension 64: 94-102, 2014.

30. Norton JD: ID helix-loop-helix proteins in cell growth, differentiation and tumorigenesis. J Cell Sci 113: 3897-3905, 2000.

31. Lasorella A, Uo T and Iavarone A: Id proteins at the cross-road of development and cancer. Oncogene 20: 8326-8333, 2001.

32. Sikder HA, Devlin MK, Dunlap S, Ryu B and Alani RM: Id proteins in cell growth and tumorigenesis. Cancer Cell 3: 525-530, 2003

33. Sun LY, Bokov AF, Richardson A and Miller RA: Hepatic response to oxidative injury in long-lived Ames dwarf mice. FASEB J 25: 398-408, 2011.

34. Martins C, Doran C, Silva IC, Miranda C, Rueff J and Rodrigues AS: Myristicin from nutmeg induces apoptosis via the mitochondrial pathway and down regulates genes of the DNA damage response pathways in human leukaemia K562 cells. Chem Biol Interact 218: 1-9, 2014.

35. Wang X, Liu Y, Shao D, Qian Z, Dong Z, Sun Y, Xing X, Cheng X, Du H, Hu Y, et al: Recurrent amplification of $M Y C$ and TNFRSF11B in $8 \mathrm{q} 24$ is associated with poor survival in patients with gastric cancer. Gastric Cancer: Jan 25, 2015 (Epub ahead of print). http://dx.doi.org/10.1007/s10120-015-0467-2.

36. Kino T, Hurt DE, Ichijo T, Nader N and Chrousos GP: Noncoding RNA gas5 is a growth arrest- and starvation-associated repressor of the glucocorticoid receptor. Sci Signal 3: ra8, 2010.

37. Mourtada-Maarabouni M, Hasan AM, Farzaneh F and Williams GT: Inhibition of human T-cell proliferation by mammalian target of rapamycin (mTOR) antagonists requires noncoding RNA growth-arrest-specific transcript s5 (GAS5). Mol Pharmacol 78: 19-28, 2010. 